\title{
Evolución de la concepción de medio ambiente en estudiantes de pedagogía en educación básica
}

\author{
Roberto Arias Arce \\ Departamento de Didáctica de la Matemática y las Ciencias Experimentales. Universitat \\ Autonoma de Barcelona. Edifici G, Plaça del Coneixement, 08193 Bellaterra (Cerdanyola \\ el Vallès), Barcelona, España. reariasa@gmail.com \\ ORCID: https://orcid.org/0000-0002-4304-5925
}

\section{Genina Calafell Subirá}

Departament d'Educació Lingüística i Literària, i Didàctica de les Ciències Experimentals i de la Matemàtica. Campus de Mundet, Universitat de Barcelona, Passeig de la Vall d'Hebron, 171, 08035 Barcelona, España. genina.calafell@ub.edu ORCID: https://orcid.org/0000-0002-4026-7207

\section{Neus Banqué Martínez}

Departamento de Didáctica de la Matemática y las Ciencias Experimentales. Universitat Autonoma de Barcelona. Edifici G, Plaça del Coneixement, 08193 Bellaterra (Cerdanyola el Vallès), Barcelona, España.neus.banque@uab.cat ORCID: https://orcid.org/0000-0003-3603-8636

[Recibido: 27 Mayo 2019. Revisado: 3 Septiembre 2019. Aceptado: 10 Diciembre 2019]

\begin{abstract}
Resumen: El presente estudio explora en la evolución de la noción de medio ambiente que despliegan estudiantes de pedagogía en educación básica chilenos, a lo largo del desarrollo de un taller constructivista en Educación Ambiental (EA), asumiendo la necesidad de actualizarla en Chile. En términos teóricos, la revisión de diversas posiciones respecto de la comprensión del medio ambiente y su identificación, mediante metodologías consideradas con la complejidad de los fenómenos ambientales, dió paso al empleo de estas en función del tipo de noción considerada, su frecuencia a lo largo del trabajo y el grado de complejidad que estas adquirieron en el tiempo. En este sentido los resultados obtenidos, dan cuenta de un desarrollo progresivo de las nociones de medio ambiente en los estudiantes, las cuales incrementan en cantidad y complejidad, condición auspiciosa ante la problemática expuesta para Chile.
\end{abstract}

Palabras clave: Cambio conceptual, Medio ambiente, Educación ambiental, Formación de Educadores.

\section{Evolution of concept of environment in students of basic education on training}

Abstract: The present study explores the evolution of the notion of the environment that Chilean pedagogy students deploy in basic education, throughout the development of a constructivist workshop on Environmental Education (EA), assuming the need to update it in Chile. In theoretical terms, the review of various positions regarding the understanding of the environment and its identification, through methodologies considered with the complexity of environmental phenomena, gave way to the use of these depending on the type of notion considered, their frequency along of work and the degree of complexity that they acquired over time. In this sense, the results obtained, show a progressive development of the notions of environment in students, which increase in quantity and complexity, an auspicious condition before the problem exposed for Chile.

Keywords: Conceptual Change, Environment, Environmental Education, Teaching training. 
Para citar este artículo: Arias Arce, R., Calafell Subirá, G. y Banqué Martínez, N. (2019). Evolución de la concepción de medio ambiente en estudiantes de pedagogía en educación básica. Revista de Educación Ambiental y Sostenibilidad $2202 . \quad 1(2), \quad$ doi: 10.25267/Rev_educ_ambient_sostenibilidad.2019.v1.i2.2202

\section{Introducción}

Vivimos en un momento de emergencia planetaria y crisis ambiental si nos atenemos a los últimos informes del Intergovernmental Panel on Climate Change (IPCC) o la declaración de 15364 científicos sobre la degradación ambiental del planeta y sus efectos (Ripple et al., 2017). Una problemática ambiental global que en Chile se encuentra alineada con el desarrollo del modelo neoliberal y la consecuente apertura hacia la instalación de empresas que desarrollarían una fuerte intervención sobre el medioambiente (Silva, 1996; Camus y Hajek, 1998).

La necesidad de impulsar y desarrollar políticas y programas educativos en favor del medio ambiente y capacitar a la ciudadanía para avanzar hacia un mundo más sostenible está consensuado internacionalmente, como lo reflejan las tres ediciones de la Cumbre de la Tierra (Estocolmo, 1972; Río de Janeiro, 1992; Johannesburgo, 2002), la Década de la Educación para el desarrollo sostenible (2004-2014) o la declaración de los objetivos para el Desarrollo Sostenible (agenda para el 2030), entre otras instancias globales.

En el contexto chileno el trabajo ambiental, a partir de la instalación de la Política Nacional de Educación para el Desarrollo Sustentable (PNEDS) y su reciente Ministerio de Medio Ambiente, asume la Educación para el Desarrollo Sostenible como el enfoque ambiental y el Sistema Nacional de Certificación Ambiental de Establecimientos Educacionales (SNCAE) como mecanismo de instalación de dichos fines (Ministerio del Medio Ambiente, 2009). De esta manera, se genera una plataforma de intervención conjunta entre instituciones públicas y comunidad educativa, así como el desarrollo de líneas de acción complementarias para fortalecer la EA, el cuidado y protección del medio ambiente y la generación de redes asociativas para la gestión ambiental local (Burgos, Perales y Gutiérrez, 2010).

En la práctica, diversos autores exponen la situación en la que se presenta la EA en las instituciones educativas chilenas; si bien es cierto se valoran los logros en gestión y en los compromisos de la comunidad educativa (Burgos, Gutiérrez y Perales, 2012), se consigna de igual forma la existencia de estrategias didácticas precarias, escasos tiempos de trabajo para el diseño y organización de actividades en el área y el desconocimiento de los fines, significado y temáticas propias de la EA en los docentes (Bravo, 2011; Torres, Benavides, Latoja, y Novoa, 2017).

De hecho, la EA que se despliega en Chile se encuentra lejos de promover una cosmovisión que considere sus principios en la construcción de futuros ciudadanos (Ponce y Vega, 2009; Muñoz, 2014), siendo necesario desarrollar transformaciones al sistema de formación de profesores, especialmente ante la realización de acciones concretas que permitan el abordaje en propiedad de la EA en nuestras aulas (Squella, 2001; Burgos, Perales y Gutiérrez, 2010).

Ante esta situación la Formación inicial de Maestros en general, y en Chile en particular, no puede obviar los retos a los que se entrenan los gobiernos y las 
instituciones, siendo urgente introducir la educación ambiental en ella y su seguimiento mediante el desarrollo de diversas investigaciones. Desde esta premisa y tomando como referencia las corrientes de educación ambiental (Sauvé, 2010), así como la investigación sobre las concepciones de medio del profesorado (Calafell y Bonil, 2014), se presenta un estudio entorno a la evolución de las nociones de medio ambiente de un grupo de estudiantes chilenos de pedagogía que participaron de un taller formativo en educación ambiental.

\section{Marco teórico}

Las aproximaciones al significado de medio ambiente y en consecuencia de EA son dinámicas y diversas. Surgida desde preocupaciones científicas y políticas sobre el estado del medio ambiente a mediados del siglo XX, la EA se caracterizó durante los años setenta por mantener las características del romanticismo naturalista, condición que dio paso en la siguiente década a desarrollar una mirada consecuente con la postmodernidad que se gestaba (Avendaño y Albores, 2014). Llegado el siglo XXI, la EA asume como objetivo la colaboración en el establecimiento de relaciones más armónicas entre los seres humanos y entre estos con su medioambiente, teniendo como fin prevenir problemas ambientales y asegurar un mundo más sano a las futuras generaciones (Terrón, 2004).

Coherente con la finalidad expuesta, una reflexión profunda surge al interior de las comunidades de educadores, sobre los elementos requeridos para potenciar la transformación de los procesos pedagógicos que se desarrollan en las aulas, hacia la consideración de la Enseñanza de las Ciencias y la EA, como elementos con alta pertinencia social en la formación y conformación de los ciudadanos del siglo XXI (Gonzales y Lárez, 2009). Entrado el siglo y producto de estas consideraciones, se plantea la posibilidad de articular ambas posiciones bajo un proyecto común, asumir la doble misión de formar a la vez los futuros profesionales de la ciencia y los ciudadanos no especialistas, pero capaces de transigir con la ciencia para la comprensión de la realidad y para la toma de decisiones y la acción (Sauvé, 2013; Chopra, 2015).

De esta forma, la presencia de diferentes movimientos ambientales que asumen la recuperación de las relaciones con el medio desde sus diversidades culturales, territoriales y productivas (Sauvé, 2010), aportan a la creación de un papel algo más amplio para la ciencia en el currículum: la reconceptualización de los objetivos de la educación científica, el papel del maestro y la pedagogía, desde las múltiples perspectivas inherentes a la educación ambiental (Chopra, 2015), promoviendo con esto una ciencia ambientalizada, pertinente y estratégica para las generaciones presentes y futuras (Mora, 2012).

\section{Relevancia y tipos de concepciones de Medio Ambiente}

Al respecto, pareciera existir sincronía en la literatura respecto de la relevancia que carga la noción de medio ambiente que asume cada docente; entendiendo que la educación ambiental no tiene una manifestación única, ni se ajusta a un prototipo exclusivo de intervención educativa característico (Gutiérrez y Pozo, 2006), la necesidad de articular diversas posiciones sobre el medio ambiente, buscando nutrir las propuestas que se aborden en el aula escolar y en los currículos formativos de los profesionales de la educación, coincide con la necesidad de transformar diferentes 
paradigmas del conocimiento, hacia la internalización de un saber ambiental complejo (Sauvé, 2010).

Al involucrar conocimientos de diferentes disciplinas, el concepto de medio ambiente presenta una complejidad con implicancias directas en su enseñanza, en la forma que cada educador conceptualiza el medio ambiente y las acciones educativas con las que vincula al alumnado con dicha noción (Calafell y Bonil, 2014), condición que requiere analizar y comparar las distintas creencias sobre el medio ambiente que se encuentran en el aula (Correa y Rodrigo, 2001).

Desde esta visión, Gutiérrez y Pozo (2006) revisa diversos modelos de educación ambiental y sus marcos teóricos de fundamentación, detallando cinco marcos teóricos que organizan los diversos modelos de educación ambiental vigentes, cada cual con un campo disciplinar y cultura científica de referencia; aspectos económicos, sociopolíticos y filosóficos son considerandos en la argumentación de tres marcos de referencia, seguidos de otros dos, asentados en aspectos pedagógicos, sociológicos y psicológicos. (Tabla 1).

Coherente con la sistematización expuesta y considerando las corrientes descritas por Sauvé (2004) (Tabla 2), Calafell y Bonil (2014), exploran las posibilidades de diagnosticar las nociones de medio ambiente de un grupo de educadores ambientales, ejercicio que los lleva a configurar tres ámbitos -Natural, Conexión y Cultural- desde los cuales dar cuenta del grado de complejidad que se presenta entre ellas. Considerando que el ámbito Natural, asume el medio ambiente como el entorno natural y como un recurso; que Conexión, lo entiende como producto de la interacción entre diversos factores; y que las nociones que incluye Cultural, toman como punto de partida a los individuos y sus relaciones con factores como la cultura, el poder, las emociones, los movimientos sociales, etc., en la construcción de una identidad ambiental individual y colectiva (Tabla 3). De esta forma, los ámbitos propuestos consideran, sin exclusiones, las diversas nociones de medio ambiente, permitiendo con esto su empleo ante la caracterización de concepciones más ricas, diversas y complejas respecto del medio ambiente (Calafell y Bonil, 2014). 
Tabla 1. Modelos de educación ambiental y sus marcos teóricos de fundamentación. Adaptado de Gutiérrez y Pozo (2006).

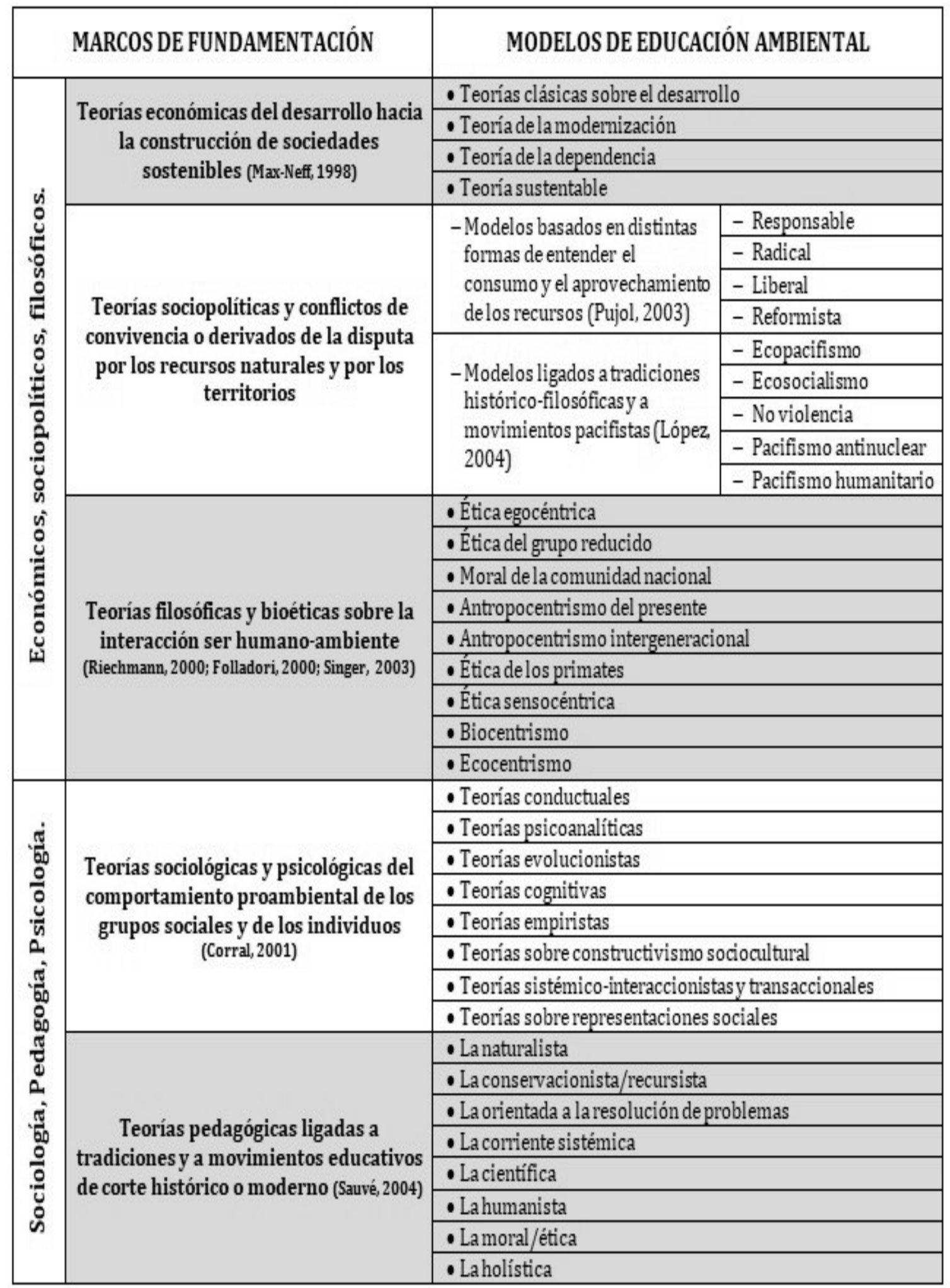


Tabla 2. Corrientes de antigua y nueva tradición en Educación Ambiental. Adaptado desde Sauvé, (2004; 2010).

\begin{tabular}{|c|c|c|c|}
\hline Tradición & $\begin{array}{c}\text { Corriente de } \\
\text { educación ambiental }\end{array}$ & $\begin{array}{l}\text { Concepción de } \\
\text { ambiente }\end{array}$ & Objetivo \\
\hline \multirow{7}{*}{ Antigua } & Naturalista & Naturaleza & Reconstruir un lazo con la naturaleza \\
\hline & $\begin{array}{l}\text { Conselvacionista/ } \\
\text { Recursista }\end{array}$ & Recurso & $\begin{array}{l}\text { Adoptar comportamientos de conservación. Desarrollar habilitidades relativas ala gestión } \\
\text { ambiental. }\end{array}$ \\
\hline & Resolutiva & Problema & Desarrollar habilidades de resolución de problemas (RP): del diagnóstico a la acción. \\
\hline & Sistémica & Sistema & $\begin{array}{l}\text { Desarrollar el pensamiento sistémico: análisis y síntesis, hacia una visión global. } \\
\text { Comprender las realidades ambientales en vista de decisiones apropiatas. }\end{array}$ \\
\hline & Cientifica & Objeto de estudio & $\begin{array}{l}\text { Addquirir conocimientos en ciencias ambientales, Desarrollar habilidades relativas a la experiencia } \\
\text { cientifica. }\end{array}$ \\
\hline & Humanista & Mediode vida & $\begin{array}{l}\text { Conocer su medio de viday conocerse mejor en relación con medio de vidar } \\
\text { Desarrollar un sentimiento de pertenencia. }\end{array}$ \\
\hline & Moral/Ética & Objeto de valores & Dar prueba de ecc-civismo, Desarrollar un sistema ético. \\
\hline \multirow{8}{*}{ Nueva } & Holística & Holos, Todo & $\begin{array}{l}\text { Desarrollar las múltiples dimensiones de su ser en interacción con el conjunto de dimensiones del } \\
\text { ambiente. Desarrollar un conocimiento "orgeganico" del mundo y un actuar participativo en y con el } \\
\text { ambiente. }\end{array}$ \\
\hline & Bio-regionalista & Lugar de pertenencia & Provecto comunitario. Desarrollar competencias en eco-desarrollo comunitario, local o regional, \\
\hline & Práxica & $\begin{array}{l}\text { Crisol de acción/ } \\
\text { reflexión }\end{array}$ & Aprender en, para y por la acción. Desarrollar competencias de reflexión. \\
\hline & Critica & $\begin{array}{l}\text { Objeto/lugarde } \\
\text { emancipación }\end{array}$ & Deconsturir las realidades sociombientales en vista de transformar lo que causa problemas \\
\hline & Feminista & Objetode solicitud & Integar los valores feministas a la relación con el ambiente \\
\hline & Etnográfica & $\begin{array}{l}\text { Lugar deidentidad } \\
\text { cultural }\end{array}$ & $\begin{array}{l}\text { Reconocer el estrecho lazo entre naturaleza y cultura. Clarificar su propia cosmologia Valorizar la } \\
\text { dimensión cultural de su relación con el ambiente. }\end{array}$ \\
\hline & Eco-educación & Crisol deidentidad & $\begin{array}{l}\text { Experimentar el medio ambiente para experimentarse y formarse eny por el ambiente, } \\
\text { Construir su relación con el mundo, con los seres otros-que-humanos. }\end{array}$ \\
\hline & $\begin{array}{l}\text { Sostenibilidad / } \\
\text { sustentabilidad }\end{array}$ & $\begin{array}{l}\text { Desarrollo social, } \\
\text { culturaly económico }\end{array}$ & $\begin{array}{l}\text { Promover un desaarollo económico respetuoso de los aspectos sociales y del ambiente. Contibuir } \\
\text { atal desarrollo. }\end{array}$ \\
\hline
\end{tabular}


Tabla 3. Comparación de las categorías de nociones de medio, consideradas por Calafell y Bonil (2014) y Sauvé (2010).

\begin{tabular}{|c|c|c|c|}
\hline \multicolumn{2}{|r|}{ Calafell y Bonil (2014) } & \multicolumn{2}{|c|}{ Sauvé (2010) } \\
\hline \multirow{2}{*}{ Natural } & Naturaleza & Naturaleza & \multirow{8}{*}{ Larga tradición } \\
\hline & Naturaleza y recursos & Recurso & \\
\hline \multirow{6}{*}{ Conexión } & Problema & Problema & \\
\hline & Red de relaciones & Sistema & \\
\hline & Objeto de estudio & Objeto de estudio & \\
\hline & Medio de vida & Medio de vida & \\
\hline & Conflicto de valores & Objeto de Valores & \\
\hline & Organismo & Holos & \\
\hline \multirow{7}{*}{ Cultural } & Espacio sociocultural & Lugar de pertenencia & \multirow{7}{*}{ Nueva tradición } \\
\hline & Espacio de actuación/reflexión & Crisol de acción/reflexión & \\
\hline & Espacio de emancipación racional & Objeto de transformación & \\
\hline & Espacio afectivo de relación de poder entre grupo & Objeto de solicitud & \\
\hline & Construcción de la identidad & Lugar de identidad & \\
\hline & Espacio de desarrollo personal. & Crisol de identidad & \\
\hline & Desarrollo económico & Recursos compartidos & \\
\hline
\end{tabular}

\section{Objetivos de investigación y metodología}

Asumiendo la necesidad de explorar las nociones de medio ambiente que cargan los estudiantes de pedagogía, como primer paso ante el desarrollo de una formación docente alineada con los principios de la EA, la presente investigación considera como finalidad: "Conocer la evolución de las nociones de medio ambiente de los estudiante de pedagogía, que participan de una actividad formativa, para determinar en qué grado influye dicha formación en sus concepciones."

Específicamente y desde esta finalidad, se han desarrollado los siguientes objetivos de investigación:

1. Identificar la cantidad y la calidad de las nociones de medio ambiente que presentan los estudiantes de pedagogía que participan de un taller formativo de educación ambiental.

2. Analizar las nociones de medio en el transcurso del taller formativo y su grado de complejidad.

\section{Contexto de la investigación}

La investigación sigue la modalidad de estudio de caso ante un grupo de estudiantes de pedagogía que participan en un taller formativo de EA, paralelo a su formación reglada. En concreto, la muestra se conforma de quince estudiantes de Pedagogía en Educación Básica con menciones (Lenguaje, Matemáticas, Ciencias Naturales, Ciencias Sociales y Primer ciclo), de la Región Metropolitana de Santiago de Chile, quienes firmaron los consentimientos informados para participar en el estudio, presentando como características comunes, cargar un primer año de formación aprobado como mínimo y con diferentes aproximaciones a la educación ambiental, según las menciones de pertenencia. 
La formación se organiza siguiendo una metodología de taller, entendiendo este como un espacio en el cual un practicante ya experimentado, adquiere mayores conocimientos o generan un producto, a través de los aportes de los demás participantes, en una organización compacta y acotada en el tiempo (Flechsig y Schiefelbein, 2003). Su empleo en la actual investigación, consideró las posibilidades de incorporar principios de educación ambiental en la epistemología docente (Mosquera, 2011) de estudiantes de pedagogía, mediante dinámicas constructivistas de trabajo, desde la modalidad espada de integración de ejes transversales en el currículum (Pujol, 2003), dadas las dificultades de ser incorporada como una actividad regular del currículum formativo de los estudiantes.

Tabla 4. Estructura de trabajo considerada en las diferentes sesiones desarrolladas en el taller.

\begin{tabular}{|c|c|c|c|c|}
\hline & Sesión 1 & Sesión 2 & Sesión 3 & Sesión 4 \\
\hline Objetivos & $\begin{array}{l}\text { Identificar las nociones de } \\
\text { medio de los asistentes. }\end{array}$ & $\begin{array}{l}\text { - Ambientalizar la } \\
\text { epistemología docente. } \\
\text { - Identificar las nociones } \\
\text { de medio de los } \\
\text { asistentes. }\end{array}$ & $\begin{array}{l}\text { - Ambientalizar la } \\
\text { epistemología docente. } \\
\text { - Identificar las nociones } \\
\text { de medio de los } \\
\text { asistentes. }\end{array}$ & $\begin{array}{l}\text { - Ambientalizar la } \\
\text { epistemología docente. } \\
\text { - Identificar las nociones } \\
\text { de medio de los } \\
\text { asistentes. }\end{array}$ \\
\hline $\begin{array}{l}\text { Estrategia } \\
\text { formativa }\end{array}$ & Exposición de temas & $\begin{array}{l}\text { Activación de ideas previas } \\
\text { y cambio conceptual }\end{array}$ & $\begin{array}{l}\text { Activación de ideas previas } \\
\text { y cambio conceptual }\end{array}$ & $\begin{array}{l}\text { Activación de ideas previas } \\
\text { y cambio conceptual }\end{array}$ \\
\hline $\begin{array}{l}\text { Resumen de } \\
\text { la dinámica }\end{array}$ & $\begin{array}{l}\text { Junto con explicar la } \\
\text { investigación y la } \\
\text { participación de los } \\
\text { asistentes, se realiza la } \\
\text { aplicación del cuestionario } \\
\text { de nociones de medio } \\
\text { ambiente }\end{array}$ & $\begin{array}{l}\text { La sesión considera el } \\
\text { trabajo con la epistemología } \\
\text { docente, a cuyo cierre se } \\
\text { aplica el cuestionario de } \\
\text { nociones de medio } \\
\text { ambiente }\end{array}$ & $\begin{array}{l}\text { La sesión considera el } \\
\text { trabajo con la epistemología } \\
\text { docente, a cuyo cierre se } \\
\text { aplica el cuestionario de } \\
\text { nociones de medio } \\
\text { ambiente }\end{array}$ & $\begin{array}{l}\text { La sesión considera el } \\
\text { trabajo con la epistemología } \\
\text { docente, a cuyo cierre se } \\
\text { aplica el cuestionario de } \\
\text { nociones de medio } \\
\text { ambiente }\end{array}$ \\
\hline $\begin{array}{c}\text { Número de } \\
\text { participantes }\end{array}$ & 15 & 15 & 13 & 9 \\
\hline $\begin{array}{l}\text { Producción } \\
\text { de los } \\
\text { estudiantes }\end{array}$ & $\begin{array}{l}\text { - Cuestionario sobre } \\
\text { nociones de medio } \\
\text { ambiente }\end{array}$ & $\begin{array}{l}\text { - Cuestionario sobre } \\
\text { nociones de medio } \\
\text { ambiente } \\
\text { - Dossier de actividades de } \\
\text { ambientalización }\end{array}$ & $\begin{array}{l}\text { - Cuestionario sobre } \\
\text { nociones de medio } \\
\text { ambiente } \\
\text { - Dossier de actividades de } \\
\text { ambientalización }\end{array}$ & $\begin{array}{l}\text { - Cuestionario sobre } \\
\text { nociones de medio } \\
\text { ambiente } \\
\text { - Dossier de actividades de } \\
\text { ambientalización }\end{array}$ \\
\hline
\end{tabular}

En este sentido, las dinámicas constructivistas abordadas se enfocaron en activar las ideas previas de los estudiantes sobre su epistemología docente (Mosquera, 2011), a través del modelo formativo de la idea vector, buscando incorporar una educación ambiental compleja en la formación de profesionales (Bonil, Calafell, Granados, Junyent y Tarín, 2012; Calafell y Junyent, 2017). Regresando al taller y como se expone en la tabla 4, se consideraron 4 sesiones de trabajo, cada una de un día de duración (8 hrs.), con objetivos y estrategias formativas específicas, las que se registraron mediante un material que cada alumno producía durante cada sesión.

\section{Diseño de la investigación}

La metodología del estudio se asienta en el paradigma cualitativo, asumiendo que la realidad es una constante construcción a partir del acuerdo entre los actores y fenómenos que se desarrollan en contextos específicos (Gutiérrez, Pozo y Fernández, 
2002; Gonzales, 2001), posición que nos abre al estudio de casos como método de investigación (Blaxter, Hughes y Tight, 2010).

La investigación toma como estrategia de obtención, tratamiento y representación de datos el trabajo desarrollado por Calafell y Bonil (2014) entorno la concepción de medio ambiente de un grupo de educadores ambientales. En este sentido la aplicación del banco de imágenes (135) propuesto por los autores, el cual se puede consultar en línea (Rizoma, s.f.), al cierre de cada una de las sesiones realizadas, permitió obtener los datos de la investigación: una imagen y un texto justificativo de las nociones de medio de cada participante para la sesión 1, 2, 3 y 4. De esta forma el proceso de tratamiento de datos asume los siguientes tiempos, propuestos por Calafell y Bonil (2014):

- Reducción del texto justificativo a segmentos de significatividad

Número Imagen: $10 T$

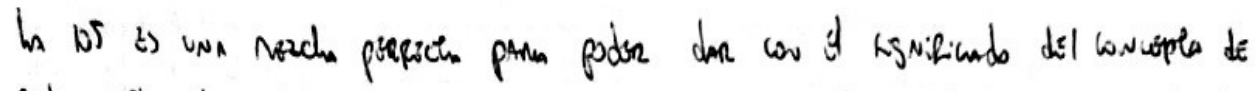

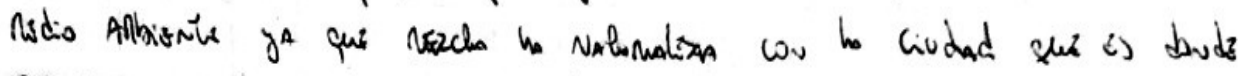

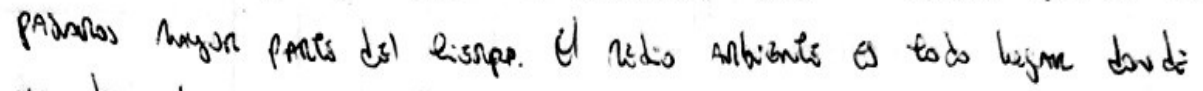

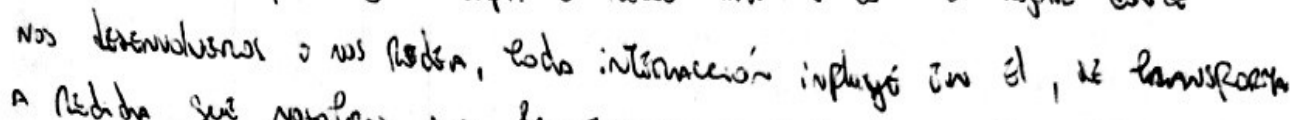

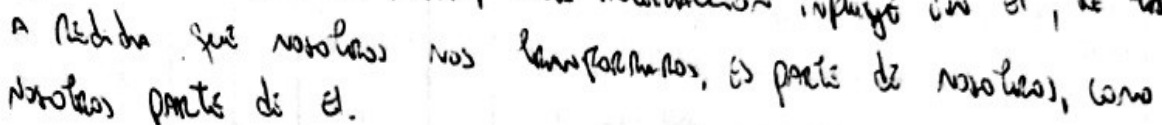

Figura 1. Texto justificativo de la asociación imagen-noción de medio ambiente expuesta por el estudiante 1 en la salida 1.

Mediante el empleo de la plantilla que expone la tabla 5, se sistematizaron las declaraciones de los estudiantes (Figura 1) a lo largo de las diferentes sesiones de trabajo.

\section{- Identificación de la(s) noción(es) de medio en el segmento de significatividad}

Tabla 5. Identificación de nociones de medio ambiente en el estudiante 1, durante la sesión 1.

\begin{tabular}{|c|c|c|c|c|}
\hline $\begin{array}{c}\mathrm{N}^{\circ} \\
\text { PART }\end{array}$ & TEXT0 & $\begin{array}{l}\text { CONCEPCIÓN MEDIO } \\
\text { AMBIENTE }\end{array}$ & $\begin{array}{l}\text { PRES (1)/ } \\
\text { AUS (0) }\end{array}$ & EVIDENCIA \\
\hline \multirow[t]{15}{*}{1} & \multirow{15}{*}{$\begin{array}{l}\text { La } 105 \text { es una mezcla perfecta para } \\
\text { poder dar con el significado del } \\
\text { concepto de medio ambiente ya que } \\
\text { mezcla la naturaleza con la ciudad que } \\
\text { es donde pasamos la mayor parte del } \\
\text { tiempo. El medio ambiente es todo } \\
\text { lugar donde nos desenvolvemos o nos } \\
\text { rodea, toda interacción influye en él, } \\
\text { se transforma a medida que nosotros } \\
\text { nos transformamos, es parte de } \\
\text { nosotros como nosotros parte de él. }\end{array}$} & Naturaleza & 0 & \\
\hline & & Recursos & 0 & \\
\hline & & Problema & 0 & \\
\hline & & Red de relaciones & 0 & \\
\hline & & Objeto estudio & 0 & \\
\hline & & Medio vida & 1 & $\begin{array}{l}\text {...para poder dar con el significado del concepto de medio } \\
\text { ambiente ya que mezcla la naturaleza con la ciudad que es } \\
\text { donde pasamos la mayor parte del tiempo }\end{array}$ \\
\hline & & Conflicto valores & 0 & \\
\hline & & Organismo & 1 & $\begin{array}{l}\text { El medio ambiente es todo lugar donde nos desenvolvemos } \\
\text { o nos rodea, toda interacción influye en él, se transforma a } \\
\text { medida que nosotros nos transformamos, es parte de } \\
\text { nosotros como nosotros parte de él. }\end{array}$ \\
\hline & & Espacio sociocultural & 0 & \\
\hline & & Espacio de actuación & 0 & \\
\hline & & Espacio de emancipación & 0 & \\
\hline & & Espacio afectivo de relación & 0 & \\
\hline & & Construcción de identidad & 0 & \\
\hline & & Espacio de desarrollo personal & 0 & \\
\hline & & Desarrollo económico & 0 & \\
\hline
\end{tabular}


Rescate de las diversas nociones de medio que se declaran en el texto justificativo, considerando la codificación de la presencia de una noción de medio ambiente con 1 y su ausencia con 0 , en la tabla 5 .

- Recuento de cada concepción de medio por participante

Considerando la codificación previa, su traspaso a la tabla 6 (ejemplificada para la sesión 1) expone la diversidad de nociones de medio que cada estudiante considera (filas) y de igual forma la frecuencia en la cual se presenta dicha noción en el conjunto de los asistentes a la actividad (columnas).

Tabla 6. Plantilla de análisis de nociones de medio ambiente.

\begin{tabular}{|c|c|c|c|c|c|c|c|c|c|c|c|c|c|c|c|c|}
\hline \multirow[t]{2}{*}{ SESIÓN 1} & \multicolumn{2}{|c|}{ Nat } & \multicolumn{6}{|c|}{ Conexión } & \multicolumn{7}{|c|}{ Cultura } & \multirow[b]{2}{*}{ 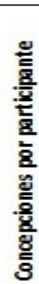 } \\
\hline & 宽 & 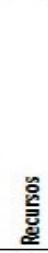 & $\begin{array}{l}\text { 폴 } \\
\text { 옿 } \\
\text { 온 }\end{array}$ & $\begin{array}{l}\text { 잏 } \\
\text { 율 } \\
\text { 올 } \\
\text { ㄹ }\end{array}$ & $\begin{array}{l}\text { 을 } \\
\text { 형 } \\
\text { 홍 } \\
\text { 옹 }\end{array}$ & $\begin{array}{l}\text { 올 } \\
\text { 욜 } \\
\text { 홀 }\end{array}$ & 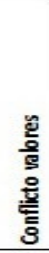 & $\begin{array}{l}\text { 을 } \\
\text { 总 } \\
\text { 总 }\end{array}$ & 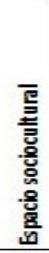 & 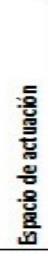 & 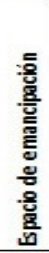 & 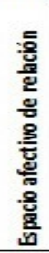 & 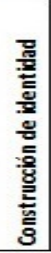 & 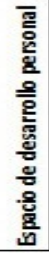 & 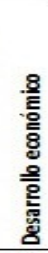 & \\
\hline 1 & 0 & 0 & 0 & 0 & 0 & 1 & 0 & 1 & 0 & 0 & 0 & o & o & o & 0 & 2 \\
\hline 2 & 0 & o & o & 1 & o & o & o & o & o & o & o & o & o & o & o & 1 \\
\hline 3 & 0 & 0 & o & $\mathbf{o}$ & o & 1 & o & o & o & o & o & o & o & o & o & 1 \\
\hline 4 & $\mathbf{o}$ & $\mathbf{0}$ & $\mathbf{0}$ & 1 & o & $\mathbf{o}$ & $\mathbf{o}$ & o & o & o & o & o & o & o & $\mathbf{o}$ & 1 \\
\hline 5 & $\mathbf{0}$ & $\mathbf{0}$ & $\mathbf{0}$ & $\mathbf{0}$ & o & 1 & $\mathbf{o}$ & o & 1 & o & o & o & o & o & $\mathbf{o}$ & 2 \\
\hline 6 & 1 & o & $\mathbf{o}$ & o & o & o & o & o & o & o & o & o & o & o & o & 1 \\
\hline 7 & o & o & o & o & o & 1 & o & o & o & o & o & o & o & o & o & 1 \\
\hline 8 & o & o & o & 1 & o & o & 1 & o & o & o & o & o & o & o & o & 2 \\
\hline 9 & 1 & o & o & o & o & o & o & o & o & o & o & o & o & o & $\mathbf{o}$ & 1 \\
\hline 10 & o & o & o & o & o & o & o & 1 & o & o & 1 & o & o & o & $\mathbf{o}$ & 2 \\
\hline 11 & $\mathbf{o}$ & o & o & 1 & o & o & o & 1 & o & o & o & o & o & o & o & 2 \\
\hline 12 & 1 & $\mathbf{0}$ & o & $\mathbf{0}$ & o & o & $\mathbf{o}$ & o & o & o & o & o & o & o & $\mathbf{o}$ & 1 \\
\hline 13 & o & o & $\mathbf{o}$ & o & $\mathbf{o}$ & 1 & $\mathbf{o}$ & o & $\mathbf{o}$ & o & o & o & o & o & o & 1 \\
\hline 14 & $\mathbf{o}$ & $\mathbf{o}$ & $\mathbf{o}$ & $\mathbf{0}$ & o & o & o & 1 & o & o & o & o & o & o & $\mathbf{o}$ & 1 \\
\hline 15 & $\mathbf{0}$ & $\mathbf{o}$ & $\mathbf{0}$ & 1 & $\mathbf{0}$ & o & o & o & o & o & o & o & o & o & $\mathbf{o}$ & 1 \\
\hline $\begin{array}{c}\text { N-de } \\
\text { concepciones } \\
\text { por corriente }\end{array}$ & 3 & o & o & 5 & o & 5 & 1 & 4 & 1 & o & 1 & o & o & o & o & \\
\hline
\end{tabular}

A partir de la información contenida en la tabla, se ha procedido a desarrollar tres estrategias de tratamiento y representación de los datos en relación a: la cantidad de las concepciones de medio ambiente por participante; el tipo de concepciones de medio ambiente por sesión y las relaciones entre la cantidad y tipo de concepciones de medio ambiente que configuran los estudiantes durante las sesiones.

- Cantidad de concepciones de medio por participante.

La realización de una tabla que recoge, por cada participante, la cantidad de concepciones que cita en cada sesión, así como el cálculo de la frecuencia y su representación en un gráfico circular. (Figura 2).
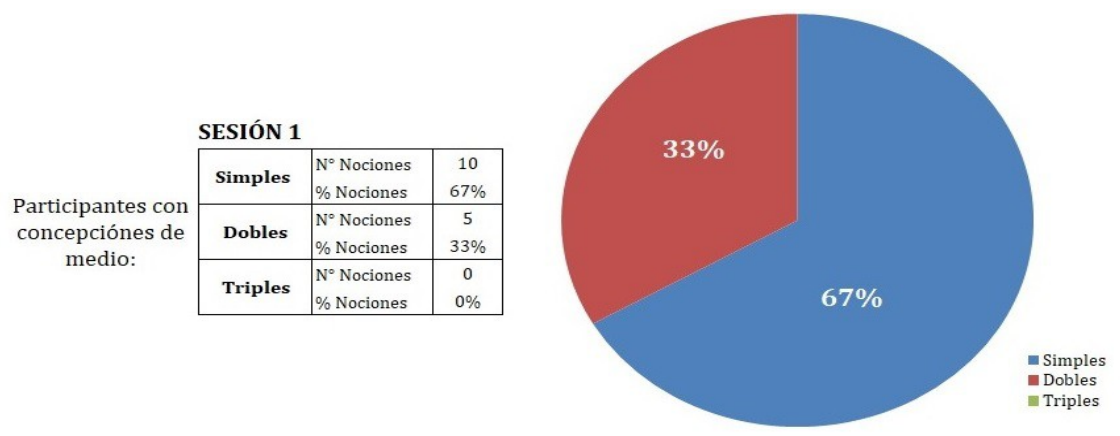

Figura 2. Cantidad de nociones de medio consideradas en la sesión 1 por los asistentes. 
- Tipo de concepciones de medio por sesión

La producción de una tabla que relaciona la cantidad total de nociones de medio ambiente que los participantes referencian, y su representación en forma de histograma a partir de los ámbitos propuestos por Calafell y Bonil (2014), nos permitirá contar con una visión total de su presencia en cada sesión de trabajo. (Figura 3).
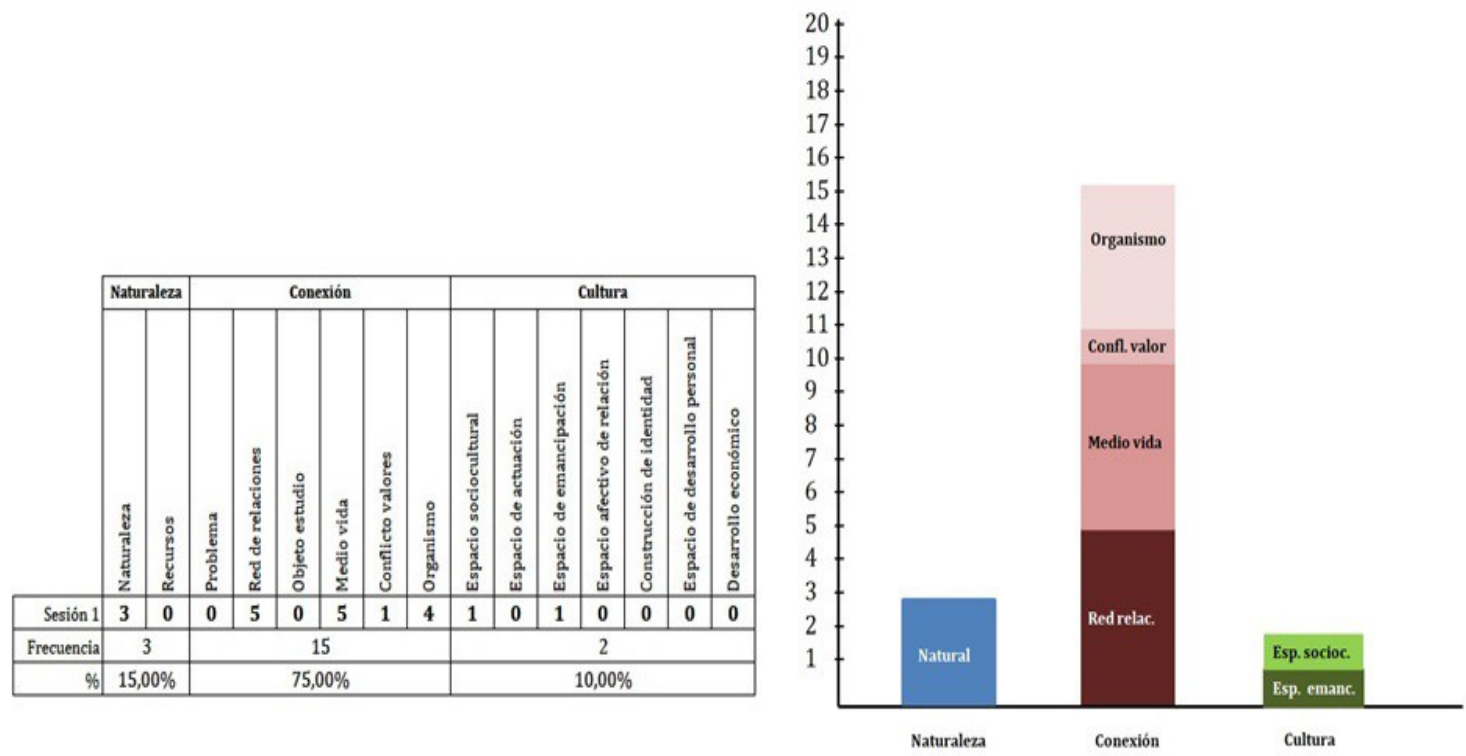

Figura 3. Diversidad de nociones de medio ambiente consideradas por los asistentes a la sesión 1.

- Relación entre cantidad y tipo de concepciones de medio consideradas

Desde las relaciones que se presentan entre los ámbitos previamente expuestos, se generaron representaciones mediante diagramas de Venn, en los cuales la cantidad de nociones que cada participante considera se expone a través de puntos de diferente grosor, mientras que el tipo de relación que se presente, se abordará a partir de la posición del participante en el centro, zona intermedia y zona externa de los círculos. (Figura 4).

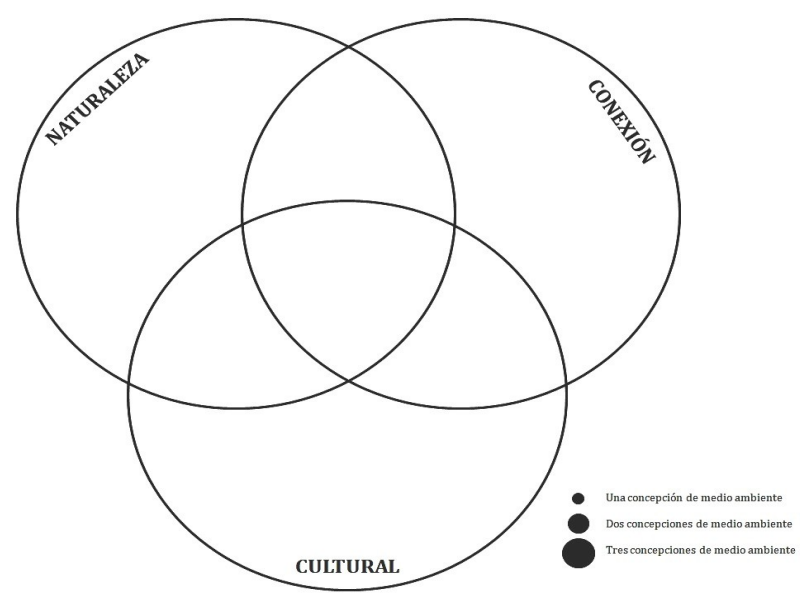

Figura 4. Diagramas de Venn para los diferentes niveles de complejidad y representación de la cantidad de nociones por puntos. Adaptado de Calafell y Bonil (2012). 
Figura 5. Niveles de complejidad en las nociones de medio ambiente (Adaptado de Calafell y Bonil, 2014).

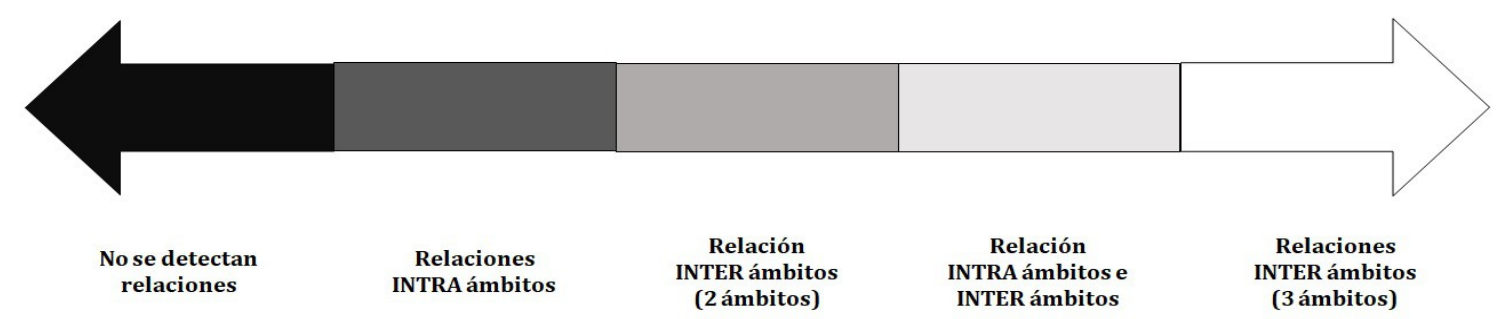

A continuación y siguiendo la propuesta de Calafell y Bonil (2014), se categorizan las relaciones que se presentan entre concepciones de medio ambiente que pertenecen a un mismo ámbito, como relaciones INTRA ámbitos, mientras que aquellas que hacen referencia a relaciones entre concepciones de medio que pertenecen a distintos ámbitos, se consideran relaciones INTER ámbitos, generando una gradiente de complejidad que va desde la inexistencia de relaciones, hasta relaciones de tipo INTER ámbitos (3 ámbitos), como máximo nivel de relaciones entre nociones de medio ambiente (Figura 5).

En la figura 6 se ejemplifica la presencia de las concepciones de medio para la sesión 1. En ésta mayoritariamente no se presentan relaciones entre concepciones y cuando se dan son relaciones intraámbitos de baja complejidad.

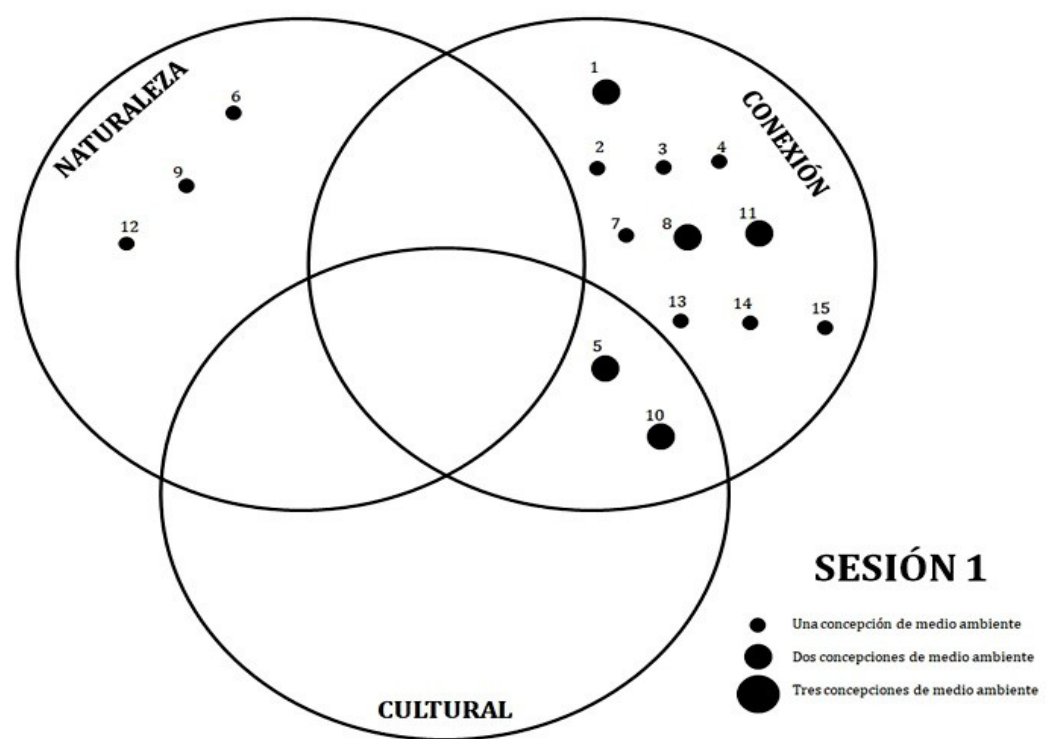

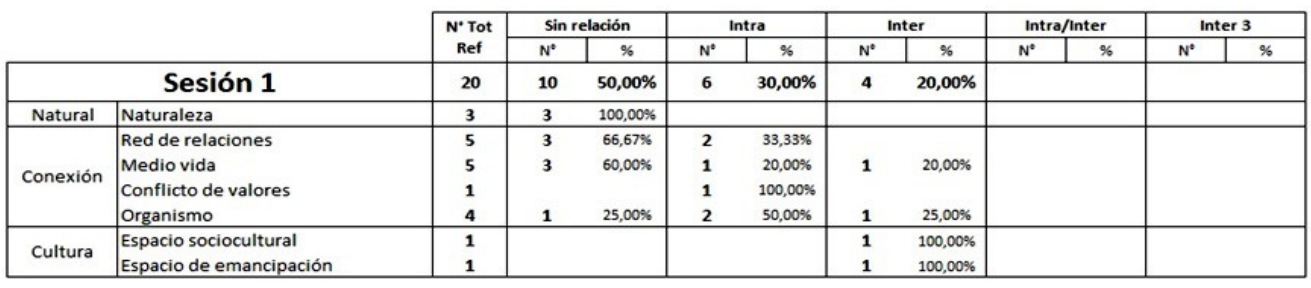

Figura 6. Nivel de complejidad en las nociones de medio expuestas por los asistentes en la sesión 1. 


\section{Resultados}

El siguiente apartado se expondrá en función de las sesiones del taller desarrollado, iniciando en cada una de ellas la caracterización de la cantidad y tipo de nociones que se generan, seguido de las relaciones que se establecen entre los ámbitos que configuran, para cerrar con el grado de complejidad que asume la sesión.

\section{Resultados sesión 1}

Los resultados de la sesión 1 (Figura 7) indican que mayoritariamente los estudiantes expresan una única concepción de medio (67\%), siendo el ámbito conexión quien agrupa las concepciones con mayor presencia (red de relaciones, medio de vida y organismo). También es un resultado a considerar que, entre las 15 concepciones propuestas por Sauvé (2004) los estudiantes asumen 7 de ellas, involucrando a todos los ámbitos. (Figura 8).
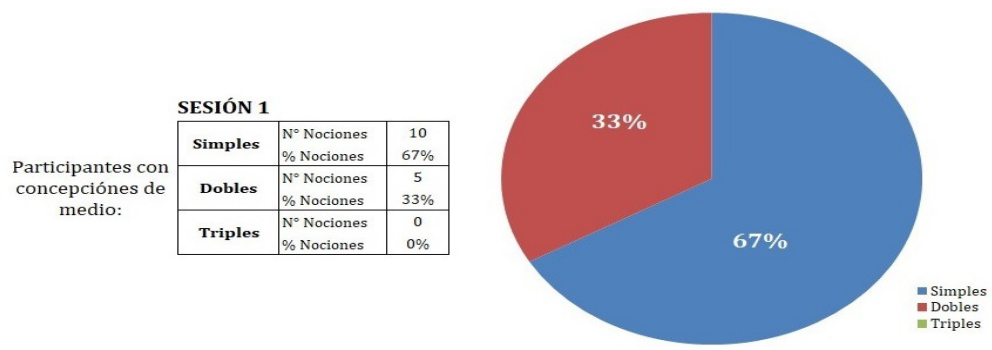

Figura 7. Cantidad de nociones de medio ambiente consideradas en la sesión 1 por los asistentes.
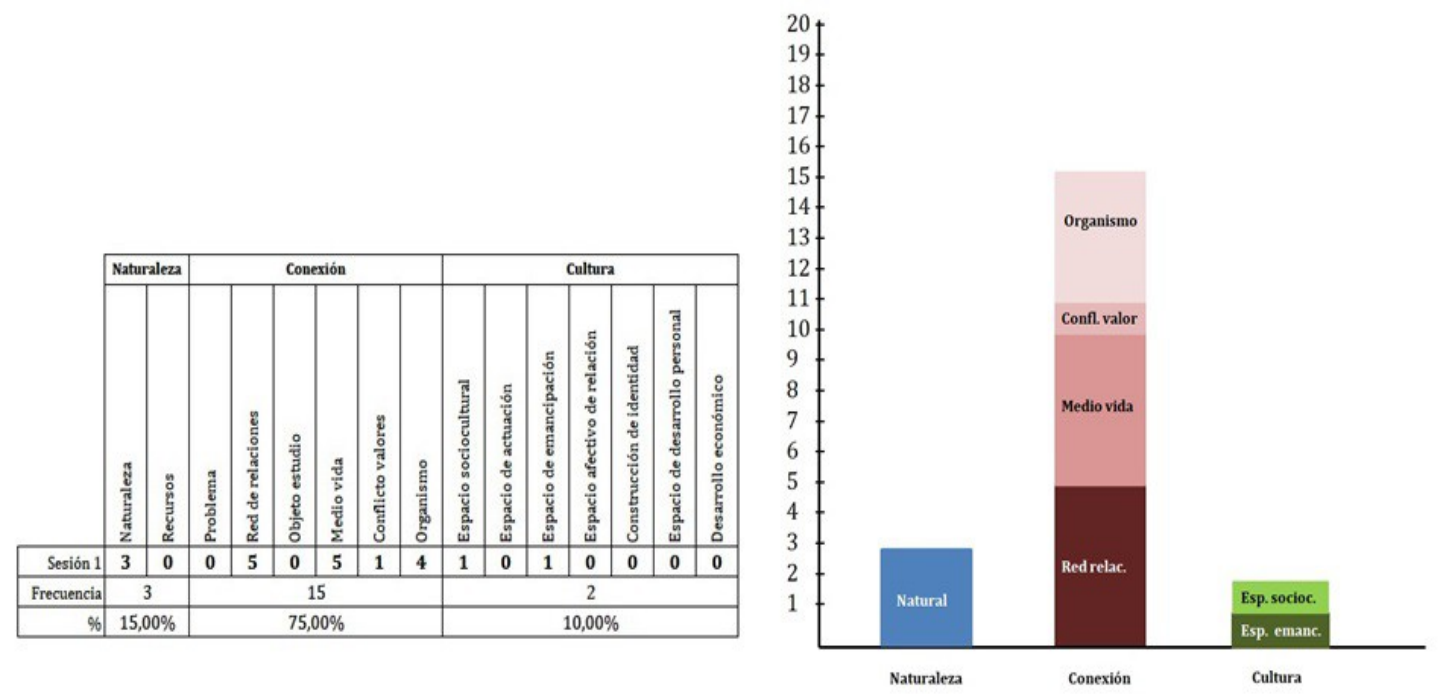

Figura 8. Diversidad de nociones de medio ambiente consideradas en la sesión 1 por los asistentes.

Respecto de las relaciones y el grado de complejidad que se presentan entre los ámbitos, la primera sesión expone un predominio de nociones aisladas (50\%), seguido de un $30 \%$ de nociones con relaciones intra ámbitos y un $20 \%$ de nociones con un grado mayor de complejidad (inter ámbitos). Específicamente, naturaleza se presenta sin relaciones; conexión distribuye sus nociones desde sin relación hasta relaciones inter ámbitos, considerando el predominio de nociones asiladas en 7 de 15 
nociones expuestas para el ámbito; y cultura, que se presenta con escasas nociones (2), todas asociadas al nivel inter de complejidad (Figura 9).

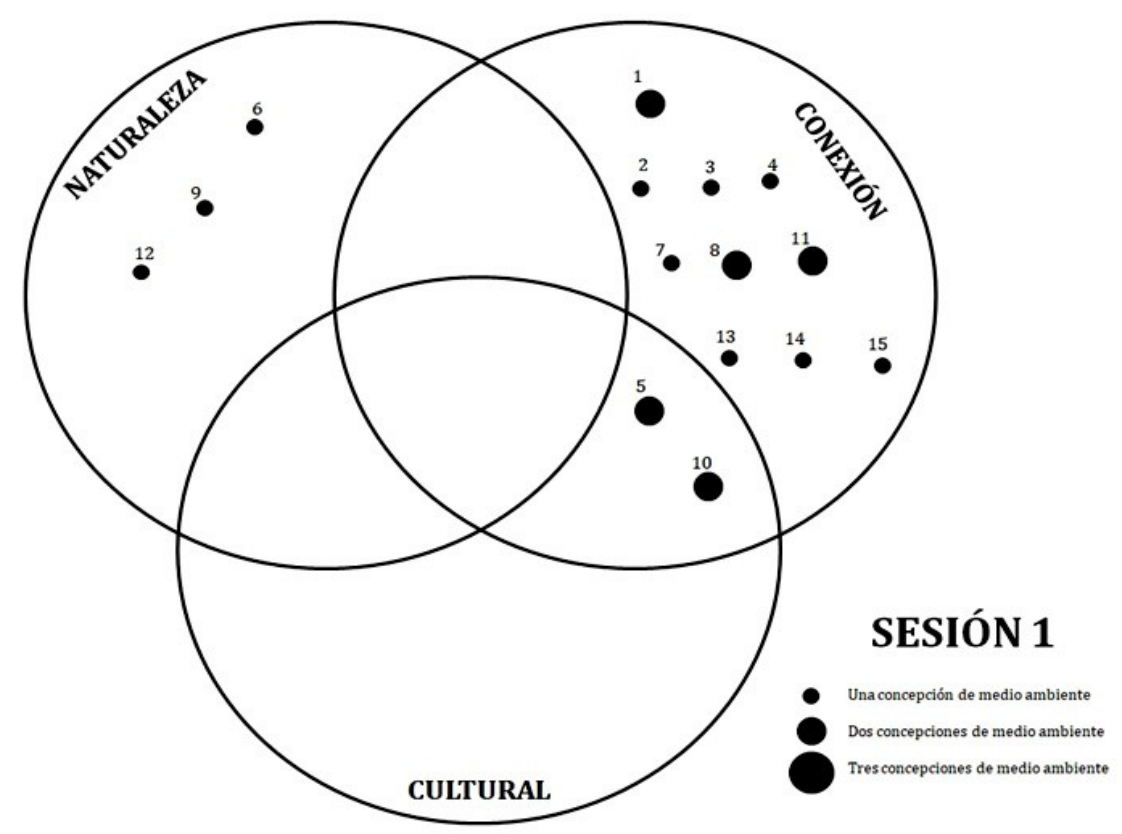

\begin{tabular}{|c|c|c|c|c|c|c|c|c|c|c|c|c|}
\hline & & \multirow{2}{*}{$\begin{array}{l}\text { N' Tot } \\
\text { Ref }\end{array}$} & \multicolumn{2}{|c|}{ Sin relación } & \multicolumn{2}{|c|}{ Intra } & \multicolumn{2}{|c|}{ Inter } & \multicolumn{2}{|c|}{ Intra/Inter } & \multicolumn{2}{|c|}{ Inter 3} \\
\hline & & & $\mathrm{N}^{*}$ & $\%$ & $\mathrm{~N}^{0}$ & $\%$ & $\mathrm{~N}^{0}$ & $\%$ & $\mathrm{~N}^{*}$ & $\%$ & $\mathrm{~N}^{*}$ & $\%$ \\
\hline \multicolumn{2}{|r|}{ Sesión 1} & 20 & 10 & $50,00 \%$ & 6 & $30,00 \%$ & 4 & $20,00 \%$ & & & & \\
\hline Natural & Naturaleza & 3 & 3 & $100,00 \%$ & & & & & & & & \\
\hline \multirow{4}{*}{ Conexión } & Red de relaciones & 5 & 3 & $66,67 \%$ & 2 & $33,33 \%$ & & & & & & \\
\hline & Medio vida & 5 & 3 & $60,00 \%$ & 1 & $20,00 \%$ & 1 & $20,00 \%$ & & & & \\
\hline & Conflicto de valores & 1 & & & 1 & $100,00 \%$ & & & & & & \\
\hline & Organismo & 4 & 1 & $25,00 \%$ & 2 & $50,00 \%$ & 1 & $25,00 \%$ & & & & \\
\hline Cultura & $\begin{array}{l}\text { Espacio sociocultural } \\
\text { Espacio de emancipación }\end{array}$ & 1 & & & & & $\begin{array}{l}1 \\
1\end{array}$ & $100,00 \%$ & & & & \\
\hline
\end{tabular}

Figura 9. Nivel de complejidad de las nociones consideradas en la sesión 1 por los asistentes.

\section{Resultados sesión 2}

Los resultados de la sesión 2 (Figura 10) indican que los estudiantes incrementan sus nociones dobles (53\%), disminuyen sus nociones sin relación (40\%) y consideran nociones triples en una ocasión (7\%). Respecto de los ámbitos considerados, naturaleza no se presenta; conexión continúa con una alta presencia, logrando un 84\% de incremento considerando las concepciones red de relaciones y medio de vida como relevantes, con ocho menciones cada una; cultura, al igual que conexión, incrementa su presencia con un 16\%, manteniendo la noción espacio sociocultural como noción relevante en las sesiones. (Figura 11). 

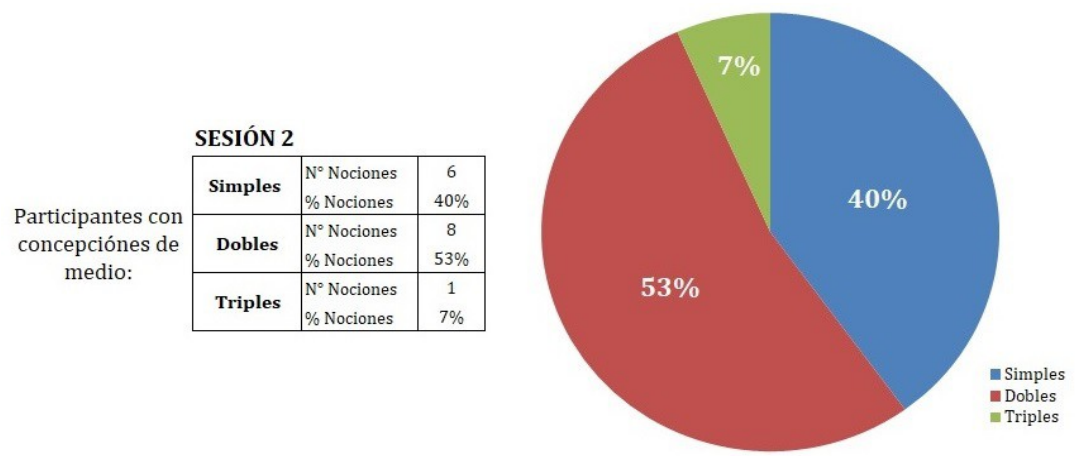

Figura 10. Cantidad de nociones de medio ambiente consideradas en la sesión 2 por los asistentes.
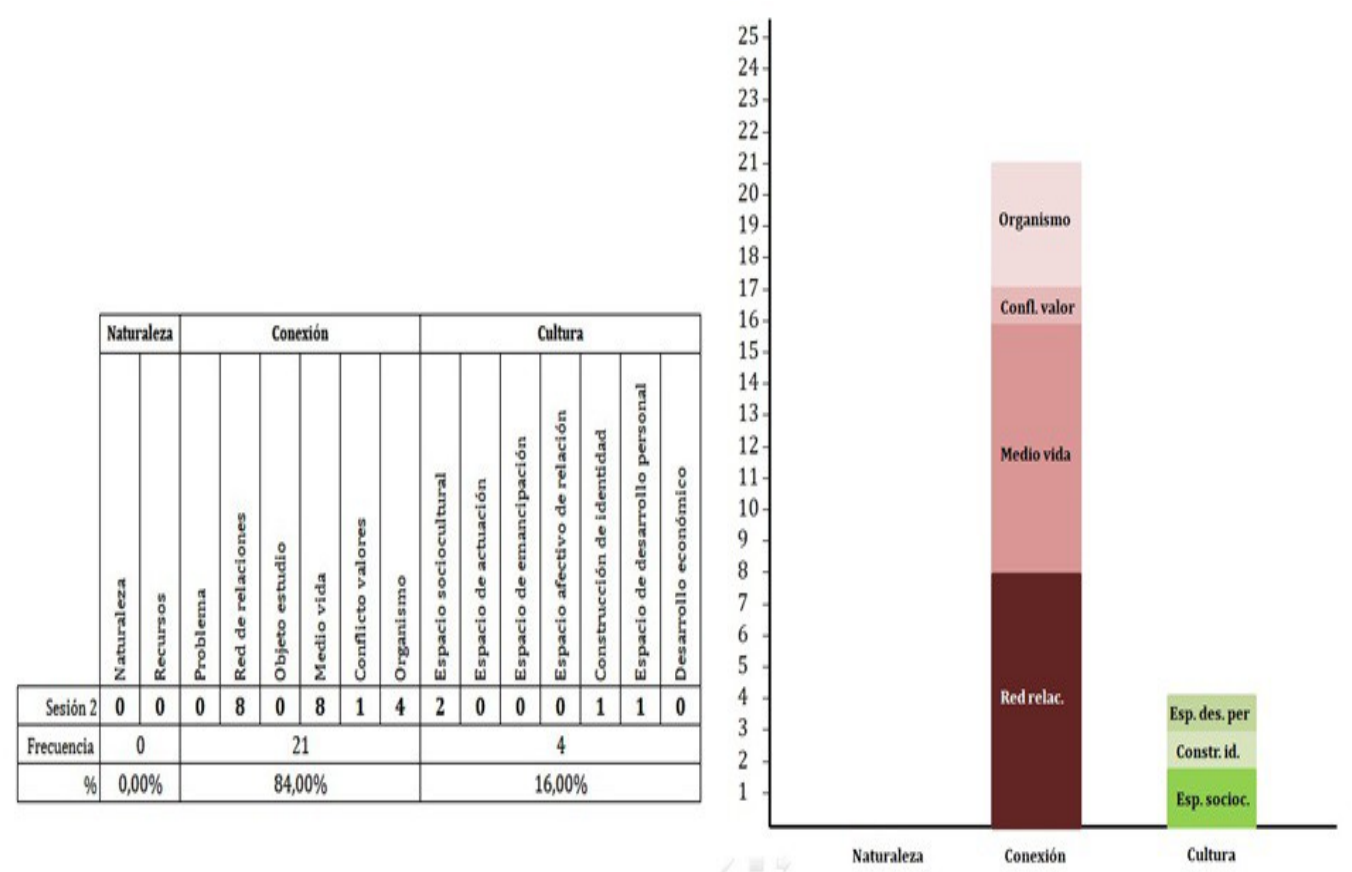

Figura 11. Diversidad de nociones de medio ambiente consideradas en la sesión 2 por los asistentes.

Respecto de las relaciones entre los ámbitos, si bien es cierto se presentan solo conexión y cultura, ambos logran el nivel inter/intra de complejidad con incipientes menciones y concentrándose en el nivel intra de relación con un 56\% de presencia en la sesión. En este sentido el incremento de frecuencias, relaciones y ámbitos considerados, permite hablar de un incremento en la percepción del medio ambiente en los asistentes a la actividad, quienes desplazan sus concepciones hacia el nivel inter/intra de complejidad (Figura 12). 


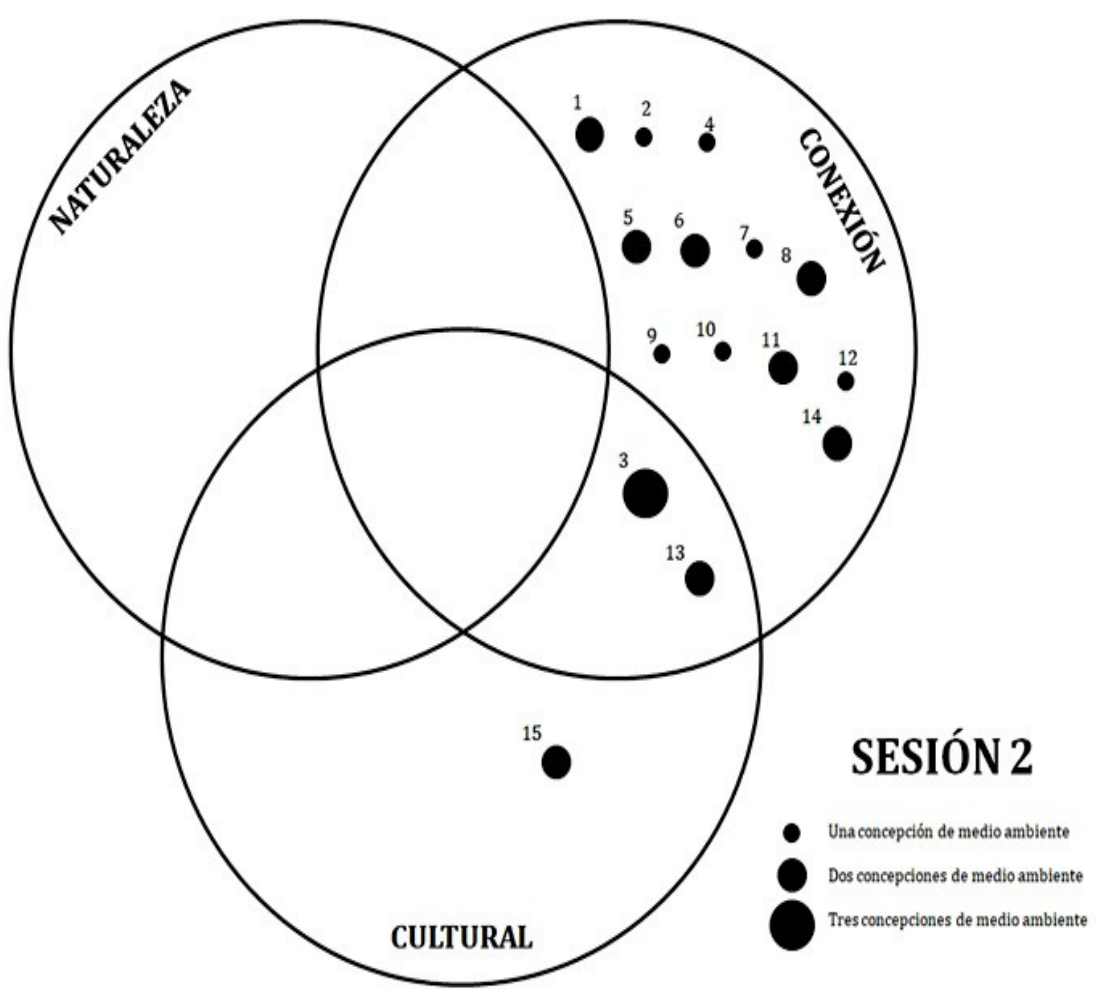

\begin{tabular}{|c|c|c|c|c|c|c|c|c|c|c|c|c|}
\hline & & \multirow{2}{*}{$\begin{array}{l}\text { N' Tot. } \\
\text { Ref. }\end{array}$} & \multicolumn{2}{|c|}{ Sin relación } & \multicolumn{2}{|c|}{ Intra } & \multicolumn{2}{|c|}{ Inter } & \multicolumn{2}{|c|}{ Intra/Inter } & \multicolumn{2}{|c|}{ Inter 3} \\
\hline & & & $\mathrm{N}^{0}$ & $\%$ & $\mathrm{~N}^{0}$ & $\%$ & $\mathrm{~N}^{0}$ & $\%$ & $\mathrm{~N}^{0}$ & $\%$ & $\mathrm{~N}^{0}$ & $\%$ \\
\hline & Sesión 2 & 25 & 6 & $24,00 \%$ & 14 & $56,00 \%$ & 2 & $8,00 \%$ & 3 & $12,00 \%$ & & \\
\hline \multirow{4}{*}{ Conexión } & Red de relaciones & 8 & 2 & $25,00 \%$ & 5 & $62,50 \%$ & & & 1 & $12,50 \%$ & & \\
\hline & Medio vida & 8 & 3 & $37,50 \%$ & 3 & $37,50 \%$ & 1 & $12,50 \%$ & 1 & $12,50 \%$ & & \\
\hline & Conflicto valores & 1 & & & 1 & $100,00 \%$ & & & & & & \\
\hline & Organismo & 4 & 1 & $25,00 \%$ & 3 & $75,00 \%$ & & & & & & \\
\hline \multirow{3}{*}{ Cultura } & Espacio sociocultural & 2 & & & 1 & $50,00 \%$ & 1 & $50,00 \%$ & & & & \\
\hline & Construcción de identidad & 1 & & & 1 & $100,00 \%$ & & & & & & \\
\hline & Espacio de desarrollo personal & 1 & & & & & & & 1 & $100,00 \%$ & & \\
\hline
\end{tabular}

Figura 12. Nivel de complejidad de las nociones consideradas en la sesión 2 por los asistentes.

\section{Resultados sesión 3}

\begin{tabular}{|c|c|c|c|}
\hline \multirow{6}{*}{$\begin{array}{l}\text { Participantes con } \\
\text { concepciónes de } \\
\text { medio: }\end{array}$} & \multicolumn{3}{|c|}{ SESIÓN 3} \\
\hline & Simples & $\begin{array}{l}N^{\circ} \text { Nociones } \\
\% \text { Nociones }\end{array}$ & $\begin{array}{c}4 \\
31 \%\end{array}$ \\
\hline & \multirow{2}{*}{ Dobles } & $N^{\circ}$ Nociones & 9 \\
\hline & & $\%$ Nociones & $69 \%$ \\
\hline & \multirow[t]{2}{*}{ Triples } & $N^{\circ}$ Nociones & 0 \\
\hline & & $\%$ Nociones & $0 \%$ \\
\hline
\end{tabular}

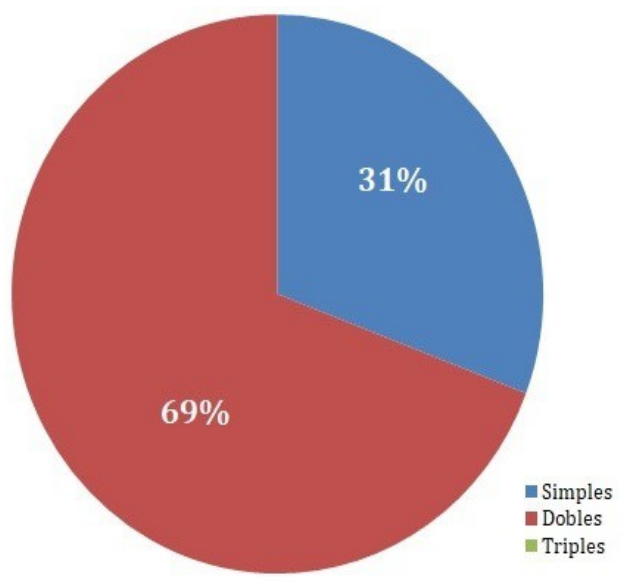

Figura 13. Cantidad de nociones de medio ambiente consideradas en la sesión 3 por los asistentes. 
Los resultados de la sesión 3 (Figura 13) muestran la inversión de las frecuencias iniciales, desde nociones simples con alta presencia (67\%) y dobles con baja presencia (33\%) hasta un 69\% nociones dobles y 30\% de nociones sin relación, sin existir comprensiones de medio ambiente con tres nociones involucradas. De esta forma, el ámbito naturaleza reaparece con una noción; conexión continúa con su alta presencia $(68,10 \%)$, manteniendo red de relaciones como tendencia relevante y enrocando organismo por medio de vida como otra noción significativa; y cultural incrementa su presencia a un 27,30\%, manteniendo las nociones espacio sociocultural y construcción de identidad como relevantes y ratificando su incremento constante en las sesiones. Finalmente, los estudiantes muestran unas concepciones que se concentran en el ámbito conexión y cultura, expresando diversidad de concepciones propuestas por Sauvé (2010), en 10 de las 15 concepciones propuestas. (Figura 14).
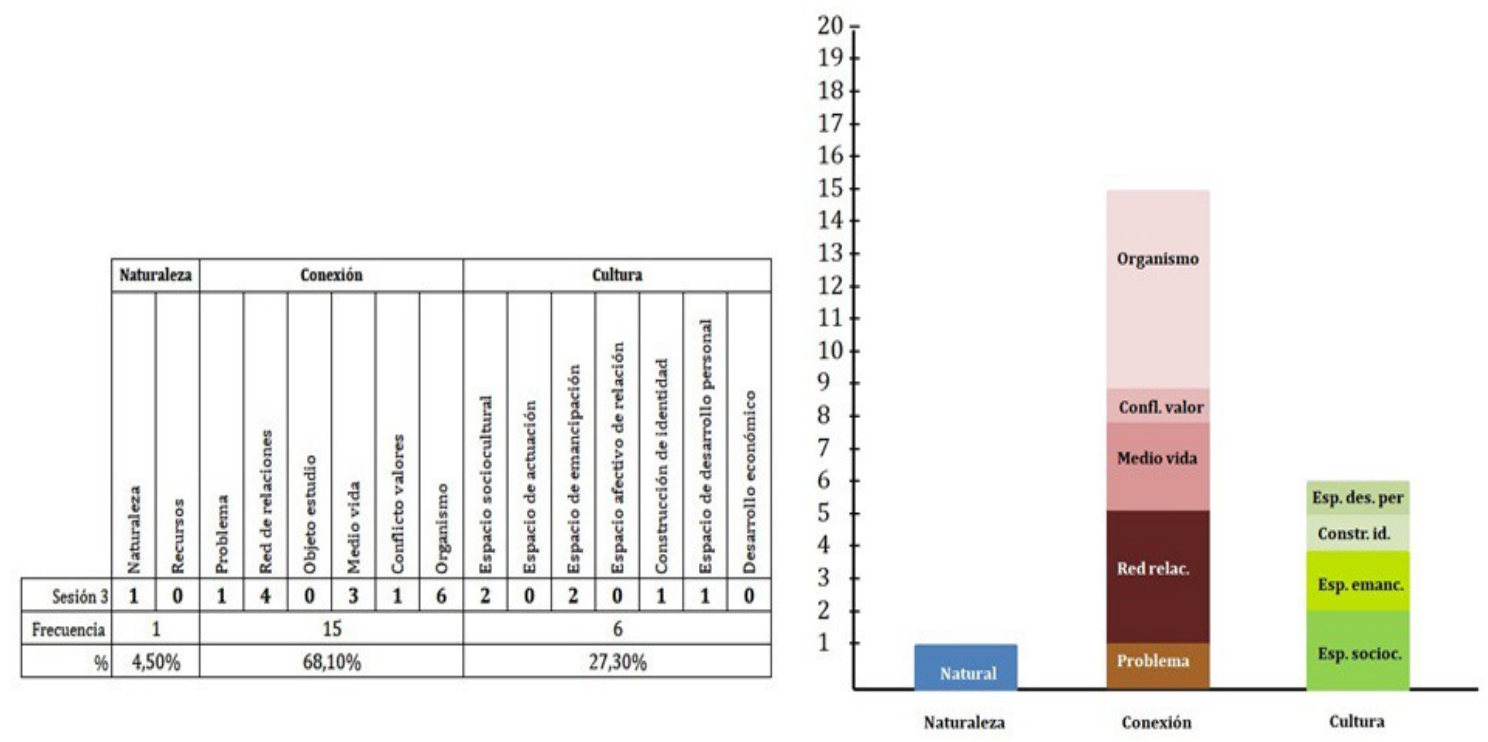

Figura 14. Diversidad de nociones de medio ambiente consideradas en la sesión 3 por los asistentes.

Detallando las relaciones que se exponen en la sesión, su concentración en el nivel intra de complejidad (54,54\%), permite dar cuenta de la fijación de las relaciones entre los ámbitos, situación que se correlaciona con la baja presencia de las nociones sin relación $(18,18 \%)$ e inter (27\%). A partir de esta condición, el grado de complejidad que adquieren las nociones de medio en los asistentes, pareciera consolidarse en su avance al primer nivel, elemento auspicioso en el trayecto hacia una complejización de la comprensión de medio ambiente que consideran los asistentes al taller (Figura 15). 


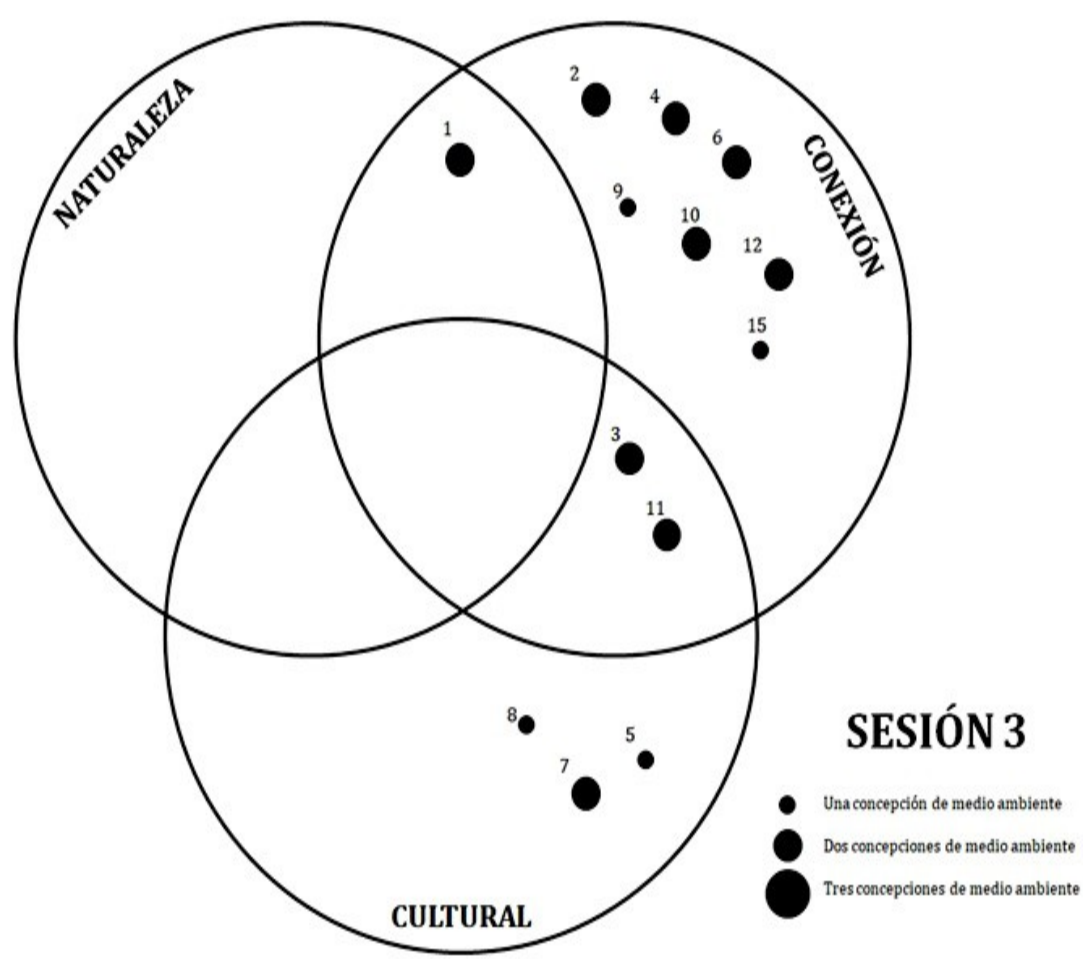

\begin{tabular}{|c|c|c|c|c|c|c|c|c|c|c|c|c|}
\hline & & \multirow{2}{*}{$\begin{array}{l}\mathrm{N}^{\prime} \text { ' Tot } \\
\text { Ref }\end{array}$} & \multicolumn{2}{|c|}{ Sin relación } & \multicolumn{2}{|c|}{ Intra } & \multicolumn{2}{|c|}{ Inter } & \multicolumn{2}{|c|}{ Intra/Inter } & \multicolumn{2}{|c|}{ Inter 3} \\
\hline & & & $\mathrm{N}^{*}$ & $\%$ & $\mathrm{~N}^{0}$ & $\%$ & $\mathrm{~N}^{0}$ & $\%$ & $\mathrm{~N}^{0}$ & $\%$ & $\mathrm{~N}^{0}$ & $\%$ \\
\hline \multicolumn{2}{|r|}{ Sesión 3} & 22 & 4 & $18,18 \%$ & 12 & $54,54 \%$ & 6 & $27,27 \%$ & & & & \\
\hline Natural & Naturaleza & 1 & & & & & 1 & $100,00 \%$ & & & & \\
\hline \multirow{5}{*}{ Conexión } & Problema & 1 & & & 1 & $100,00 \%$ & \multirow{5}{*}{$\begin{array}{l}1 \\
2\end{array}$} & \multirow{5}{*}{$\begin{array}{r}100,00 \% \\
33,33 \%\end{array}$} & & & & \\
\hline & Red de relaciones & 4 & & & 4 & $100,00 \%$ & & & & & & \\
\hline & Medio vida & 3 & 1 & $33,33 \%$ & 2 & $66,67 \%$ & & & & & & \\
\hline & Conflicto de valores & 1 & & & & & & & & & & \\
\hline & Organismo & 6 & 1 & $16,67 \%$ & 3 & $50,00 \%$ & & & & & & \\
\hline \multirow{4}{*}{ Cultura } & Espacio sociocultural & 2 & \multirow{4}{*}{$\begin{array}{l}1 \\
1\end{array}$} & \multirow{4}{*}{$\begin{array}{c}50,00 \% \\
100,00 \%\end{array}$} & 1 & $50,00 \%$ & 1 & $50,00 \%$ & & & & \\
\hline & Espacio de emancipación & 2 & & & & & \multirow[t]{3}{*}{1} & \multirow[t]{3}{*}{$50,00 \%$} & & & & \\
\hline & Construcción de identidad & 1 & & & & & & & & & & \\
\hline & Espacio de desarrollo personal & 1 & & & 1 & $100,00 \%$ & & & & & & \\
\hline
\end{tabular}

Figura 15. Nivel de complejidad de las nociones consideradas en la sesión 3 por los asistentes.

\section{Resultados sesión 4}

Los resultados de la sesión 4 (Figura 16) indican que más del 78\% de los estudiantes expresan su noción de medio considerando dos o tres concepciones, siendo las nociones dobles quienes mantienen su presencia (68\%) y las nociones triples reaparecen con una selección (11\%). En relación con el tipo de concepciones de medio que expresan los estudiantes, estas continúan centradas en conexión (70\%) considerando red de relaciones y organismo como nociones relevantes; de igual forma, cultura mantiene la presencia de espacio sociocultural y construcción de identidad como nociones significativas dada su continuidad en las sesiones, incluyendo en esta ocasión a tres nociones más del ámbito logrando una presencia del $23,50 \%$, y ratificando su tendencia a incrementar en las selecciones de los estudiantes. Finalmente el ámbito natural ratifica la presencia exclusiva de su noción homologa, así como su baja presencia en el taller (una mención, 5,9\%). (Figura 17) 

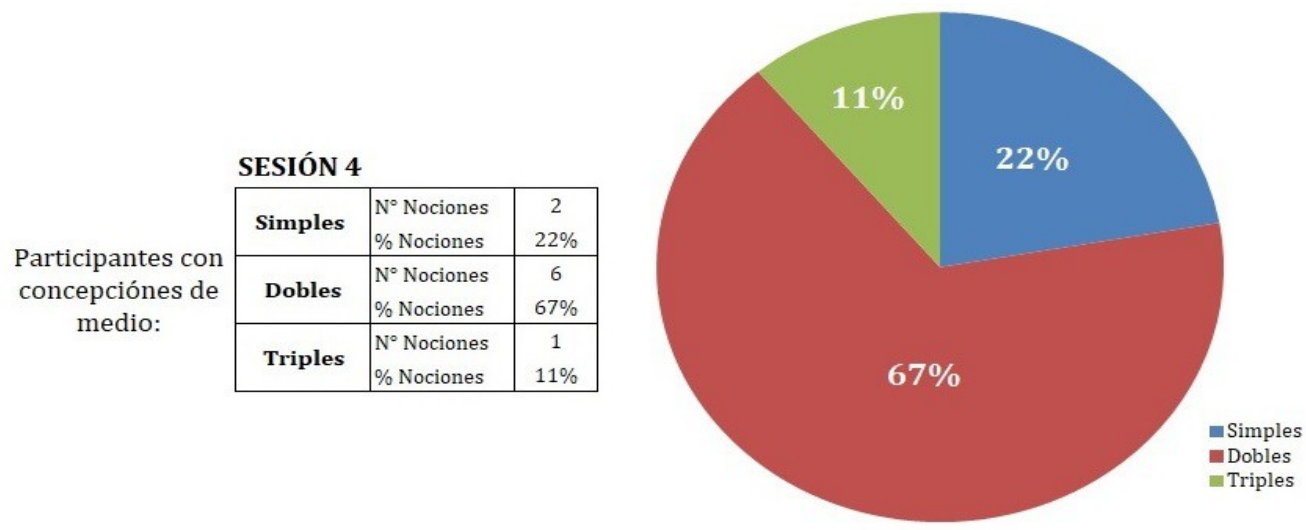

Figura 16. Cantidad de nociones de medio ambiente consideradas en la sesión 4 por los asistentes.

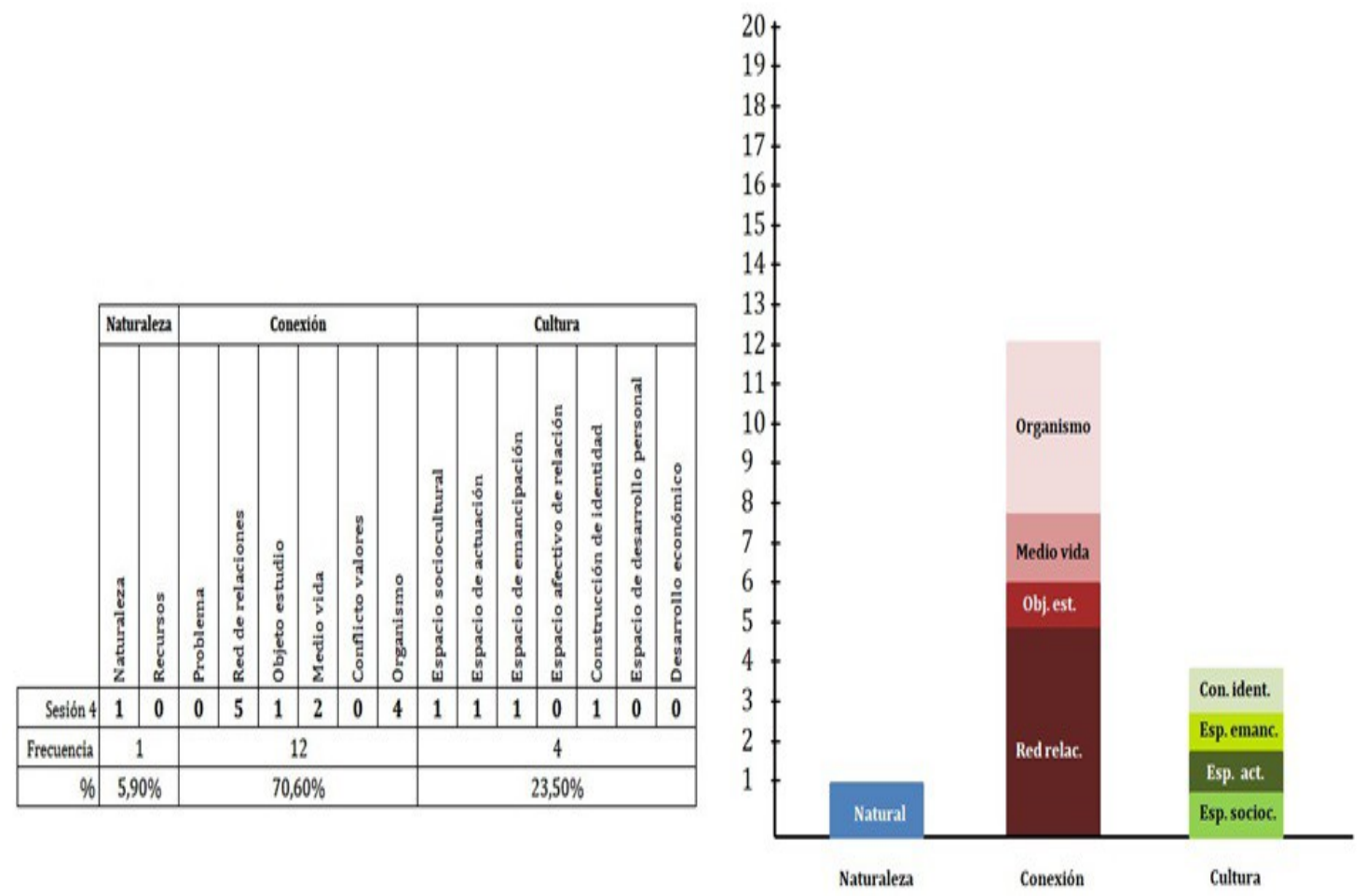

Figura 17. Diversidad de nociones de medio ambiente consideradas en la sesión 4 por los asistentes.

Respecto de la complejidad de la sesión, si bien es cierto continúa presente el nivel inter de complejidad, aglomerando las relaciones presentes en la sesión (47,05\%), se detecta una desplazamiento de estas hacia nociones de tipo intra/inter (17,64\%), así como una disminuida presencia de nociones sin relación $(11,78 \%)$ e intra $(23,52 \%)$. En este sentido y articulando los resultados de la última sesión de trabajo, pese a la baja asistencia de estudiantes, se evidencia el incremento en el grado de complejidad que se considera, coherente con el desplazamiento entre los diferentes ámbitos, lográndose avances significativos hacia niveles altos de complejidad. (Figura 18). 


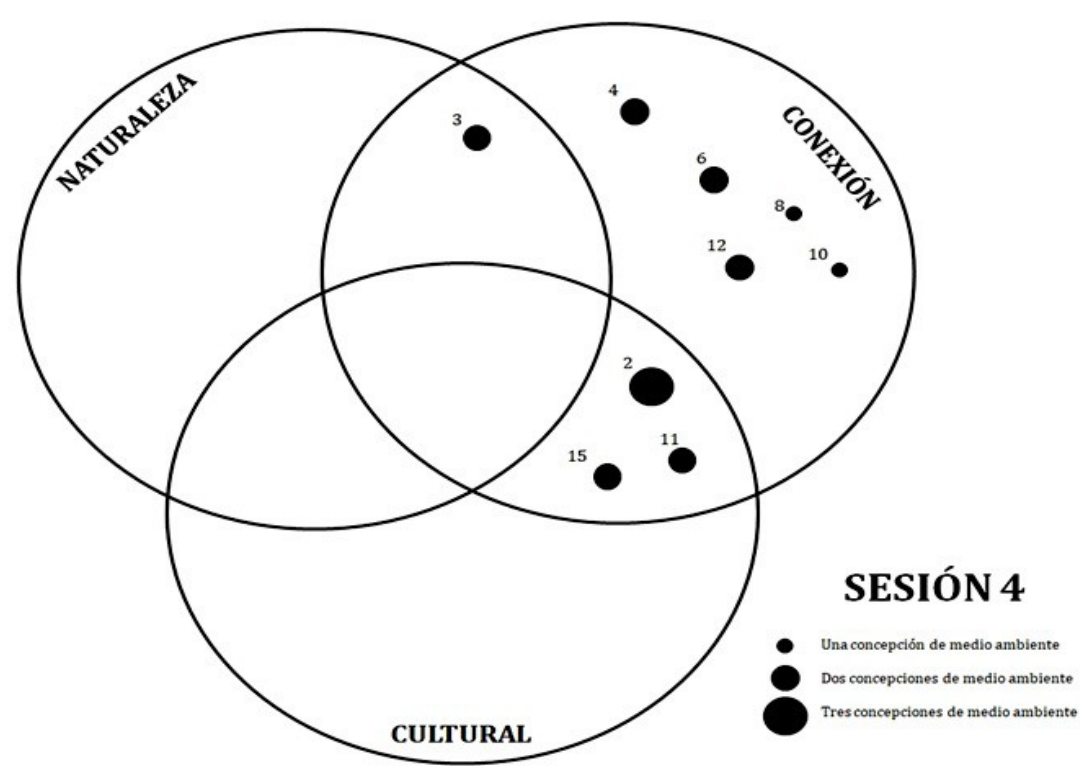

\begin{tabular}{|c|c|c|c|c|c|c|c|c|c|c|c|c|}
\hline & & \multirow{2}{*}{$\begin{array}{c}\mathbf{N}^{*} \text { Tot } \\
\text { Ref }\end{array}$} & \multicolumn{2}{|c|}{ Sin relación } & \multicolumn{2}{|c|}{ Intra } & \multicolumn{2}{|c|}{ Inter } & \multicolumn{2}{|c|}{ Intra/Inter } & \multicolumn{2}{|c|}{ Inter 3} \\
\hline & & & $\mathrm{N}^{\circ}$ & $\%$ & $\mathrm{~N}^{\circ}$ & $\%$ & $\mathrm{~N}^{*}$ & $\%$ & $\mathrm{~N}^{0}$ & $\%$ & $\mathrm{~N}^{\bullet}$ & $\%$ \\
\hline \multicolumn{2}{|r|}{ Sesión 4} & 17 & 2 & $11,76 \%$ & 4 & $23,52 \%$ & 8 & $47,05 \%$ & 3 & $17,64 \%$ & & \\
\hline Natural & Naturaleza & 1 & & & & & 1 & $100,00 \%$ & & & & \\
\hline \multirow{4}{*}{ Conexión } & Red de relaciones & 5 & & & 3 & $60,00 \%$ & 1 & $20,00 \%$ & 1 & $20,00 \%$ & & \\
\hline & Objeto de estudio & 1 & & & & & 1 & $100,00 \%$ & & & & \\
\hline & Medio vida & 2 & 1 & $50,00 \%$ & & & 1 & $50,00 \%$ & & & & \\
\hline & Organismo & 4 & 1 & $25,00 \%$ & 1 & $25,00 \%$ & 2 & $50,00 \%$ & & & & \\
\hline \multirow{4}{*}{ Cultura } & Espacio sociocultural & 1 & & & & & & & 1 & $100,00 \%$ & & \\
\hline & Espacio de actuación & 1 & & & & & & & 1 & $100,00 \%$ & & \\
\hline & Espacio de emancipación & 1 & & & & & 1 & $100,00 \%$ & & & & \\
\hline & Construcción de identidad & 1 & & & & & 1 & $100,00 \%$ & & & & \\
\hline
\end{tabular}

Figura 18. Nivel de complejidad de las nociones consideradas en la sesión 4 por los asistentes.

\section{Resultados de las cuatro sesiones}

Las concepciones de medio que expresan los participantes tienden a variar a lo largo del taller formativo, en términos cuantitativos y cualitativos. La frecuencia en que se presentan las quince concepciones de medio propuestas por Sauvé (2010), aumenta a partir de la mitad del taller (Tabla 7), resultado que se complementa con la evolución cualitativa, considerando la presencia de una concepción de medio por estudiante al inicio del taller (67\% de nociones simples en la sesión 1), pasando a consolidar la presencia de dos concepciones de medio a partir de la sesión 2 (53\% sesión 2, 69\% sesión 3 y 67\% sesión 4). (Tabla 8).

Tabla 7. Trasformaciones cuantitativas de nociones de medio ambiente consideradas.

\begin{tabular}{ccc}
\hline \multicolumn{3}{c}{$\begin{array}{c}\text { Cantidad de concepciones } \\
\text { presentes por sesion }\end{array}$} \\
\hline Sesion 1 & 7 de 15 & $46,67 \%$ \\
Sesion 2 & 7 de 15 & $46,67 \%$ \\
Sesion 3 & 10 de 15 & $66,67 \%$ \\
Sesion 4 & 9 de 15 & $60,00 \%$ \\
\hline
\end{tabular}


Tabla 8. Transformaciones cualitativas de las nociones de medio ambiente consideradas.

\begin{tabular}{|c|cc|c|c|c|c|c|c|}
\hline \multirow{2}{*}{$\begin{array}{c}\mathbf{N}^{\circ} \text { nociones de } \\
\text { medio }\end{array}$} & \multicolumn{2}{|c|}{ Sesion 1 } & \multicolumn{2}{|c|}{ Sesión 2 } & \multicolumn{2}{c|}{ Sesión 3 } & \multicolumn{2}{c|}{ Sesión 4 } \\
\cline { 2 - 9 } & $\mathbf{n}^{\circ}$ & $\mathbf{\%}$ & $\mathbf{n}^{\circ}$ & $\mathbf{\%}$ & $\mathbf{n}^{\circ}$ & $\mathbf{\%}$ & $\mathbf{n}^{\circ}$ & $\mathbf{\%}$ \\
\hline 1 & 10 & $\mathbf{6 7 \%}$ & 6 & $\mathbf{4 0 \%}$ & 4 & $\mathbf{3 1 \%}$ & 2 & $\mathbf{2 2 \%}$ \\
2 & 5 & $\mathbf{3 3} \%$ & 8 & $\mathbf{5 3} \%$ & 9 & $\mathbf{6 9 \%}$ & 6 & $\mathbf{6 7 \%}$ \\
3 & 0 & $\mathbf{0 \%}$ & 1 & $\mathbf{7 \%}$ & 0 & $\mathbf{0 \%}$ & 1 & $\mathbf{1 1 \%}$ \\
\hline Tot. & 15 & $\mathbf{1 0 0} \%$ & 15 & $\mathbf{1 0 0} \%$ & 13 & $\mathbf{1 0 0} \%$ & 9 & $\mathbf{1 0 0 \%}$ \\
\hline
\end{tabular}

Resultados que indican que la formación recibida por los estudiantes contribuye a diversificar sus concepciones de medio, infiriéndose en los participantes aproximaciones a la educación ambiental más diversas. En este sentido, estudios previos (Rivero, Martín del Pozo, Solís, Azcárate y Porlán, 2017) coinciden en señalar que los estudiantes, cuando inician un curso formativo, mayoritariamente tienen modelos educativos similares y homogéneos, situación que en su desarrollo evolucionan hacia diferentes itinerarios de concebir la educación. De este modo, resulta significativo considerar que si los maestros tienen más de una concepción de medio ambiente, no son reduccionistas, situación que les permitiría adaptarse al contexto y a los propósitos de la EA, mediante el empleo de estrategias didácticas pertinentes.

En relación al tipo de nociones que se consideran, desde los ámbitos surge con claridad el ámbito conexión como relevante, asociado a red de relaciones como noción matriz, la cual se acompaña de medio de vida en las dos primeras sesiones, para luego hacerlo con organismo en las dos siguientes. En el caso del ámbito cultura, su presencia resulta más bien baja en las dos primeras sesiones (inferior al $20 \%$ de presencia), incrementando en las dos sesiones restantes y exponiendo a la noción espacio sociocultural como relevante, seguida de construcción de identidad y espacio de emancipación, como segunda presencia constante en las sesiones. Finalmente el ámbito naturaleza presenta una escasa presencia, la cual no pasa del $10 \%$ en promedio durante las sesiones, centrada exclusivamente en la noción naturaleza. (Figura 19).

A partir de lo expuesto, resulta relevante considerar que si bien las concepciones varían su presencia en las distintas sesiones, se detectan dos grupos de nociones; aquellas estructurantes y permanentes, como red de relaciones y espacio sociocultural, y aquellas que varían o complementan a las anteriores a lo largo de las sesiones, como organismo o construcción de identidad. En este sentido y considerando el trabajo de Calafell y Bonil (2014), se refrenda la presencia del ámbito conexión con alta cantidad de nociones iniciales, las cuales progresivamente dialogan con otros ámbitos, ratificándolo como un ámbito con identidad propia. Por otra parte y a diferencia de los autores considerados (Calafell y Bonil, 2014), el ámbito naturaleza no se presenta con igual peso en las consideraciones de los asistentes, dando cuenta de un bajo grado de tradicionalismo en las concepciones expuestas por el actual grupo. 


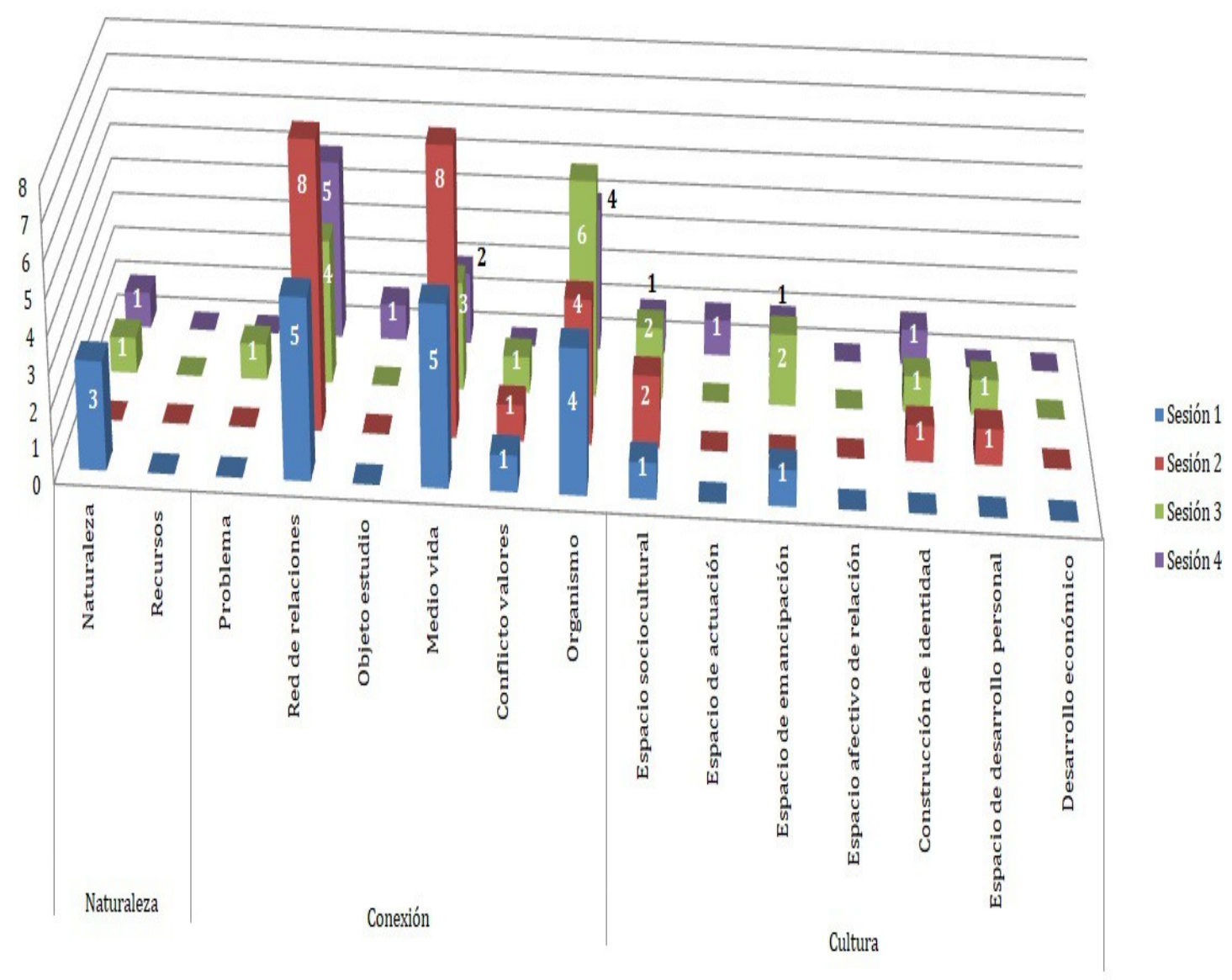

Figura 19. Nociones de medio consideradas, en la totalidad de las sesiones de trabajo, por los asistentes a la actividad.

Desde esta posición y en relación a la ausencia de algunas nociones, ambos estudios comparten la baja o nula presencia de la noción recurso, objeto de estudio, espacio de actuación, espacio afectivo de relación y desarrollo económico, exponiendo con esto la desconsideración del medio como proveedor de recursos, así como una escasa transdisciplinariedad y visión de género en la comprensión del medio ambiente que cargan los participantes de ambos contextos.

Respecto de la complejización de las nociones de medio ambiente de los asistentes, resulta plausible exponer un incremento progresivo en el nivel de complejidad que adquieren, el cual va desde altas frecuencias (50\%) para nociones sin relación en la primera sesión, hasta similar condición (47\%) en relaciones inter ámbitos en la cuarta sesión, llegando al nivel de relación inter/intra como máximo grado de complejidad entre las sesiones trabajadas, pero con bajas frecuencias $(17,64 \%)$. Al respecto, resulta relevante considerar los aportes generado por Rodríguez, Fernández y García (2014), quienes mediante la hipótesis de progresión, enfatizan en la recursividad de los procesos formativos, rehuyendo de la linealidad; la noción de sistemas de ideas que interactúan entre sí, incluyéndose unas en otras, según su mayor o menor grado de generalidad (Rodríguez, Fernández y García, 2014), nos lleva a considerar las migraciones que se presentan entre las diferentes nociones de medio que se trabajan. 
En este sentido llama la atención la migración de las frecuencias entre las sesiones 1 y 3 , de nociones sin relación a relaciones "inter", acompañada de la desagregación de las frecuencias en las sesiones 2 y 4, entre "sin relación" e "inter/intra", permitiendo postular un ciclo de contracción-distensión en el desarrollo de la complejidad en las nociones de medioambiente de los participantes. De esta forma, pareciera ser que el taller abre la posibilidad de debatir y socializar nuevas posturas sobre el entorno, situación que resulta coherente con una apertura en sus nociones iniciales, hacia su posterior diversificación.

Para concluir, existen algunas recurrencias que permiten concluir, como características iniciales de las nociones de medio ambiente:

- Su presencia sin relación con otras nociones en más de la mitad de los asistentes, y dobles en uno de cada tres sujetos indagados

- La selección inicial de 7 u 8 nociones de medio ambiente, en su mayoría pertenecientes al ámbito conexión (red de relaciones y organismo) y la ausencia de otras (recursos y espacio afectivo de relación) en la totalidad de las sesiones

- La tendencia de los ámbitos cultural y natural, de participar en relaciones de mayor complejidad (intra ámbitos, inter ámbitos e intra/inter ámbitos), así como para el ámbito conexión de ser el núcleo desde el cual se conciben la diversidad de nociones de medio ambiente.

\section{Conclusiones}

Desde estas regularidades y considerando el contexto formativo en el que se sitúa la experiencia, resulta relevante considerar el cambio de concepciones a partir de una actividad como un taller constructivista, lo cual promueve la evolución de un concepto de medio ambiente reduccionista, a una concepción más abierta y dinámica de este, facilitándose una educación ambiental menos tradicional.

En este sentido, una formación en educación ambiental desde una perspectiva transversal y compleja ayuda a cambiar las concepciones de medio ambiente y por ende de la EA, desde una visión homogénea a una visión heterogénea, situación que nos abre a preguntamos si los asistentes serán mejores profesores, dada su potencial adaptación al contexto, o bien si contribuirán a generar alumnos con mayores aproximaciones a lo que significa educar en y para el medio ambiente, en especial considerando las demandas globales y locales existentes ente la actualización y desarrollo de la educación ambiental.

De hecho, si nos situamos en un contexto persistente en visiones tradicionalistas, arraigadas en la entrega de contenidos acabados y verdaderos, como es el caso chileno, resulta relevante poder responder, ¿qué formación deberíamos incluir en los programas de formación inicial de maestros y para qué? Si queremos una educación más global y holística, debemos utilizar nuevos enfoques educativos, permitiendo que los estudiantes evolucionen sus concepciones (en nuestro caso de medio ambiente), desarrollen nuevas dinámicas de trabajo y enfrenten nuevos problemas, características que la perspectiva de la educación ambiental y la complejidad abren como una posibilidad. 
En nuestro rol de formadores de las futuras generaciones de educadores, no podemos perder de vista que situaciones como la que se presentan en Chile ante la EA -con escasas estrategias didácticas, tiempos de trabajo y definiciones en torno a los fines del movimiento-, nos deben abrir a nuevas posiciones y formas de modificar las creencias e ideas que cargan nuestros estudiantes sobre el tema, en especial dados los antecedentes que se presentan en el área. De esta forma, si la EA tiene poder transformador y se aprende, debería contemplarse en los planes de la formación de maestros, como un marco de empoderamiento y transformación social. La EA puede ser un elemento clave, no como un elemento opcional, complementario, más bien como un elemento central, con capacidad de cambio, transversal y esencial en los planes de formación inicial de maestros.

\section{Referencias bibliográficas}

Avendaño, V. y Albores, I. (2014). La educación ambiental. Entre la modernidad y la posmodernidad. Revista Caribeña de Ciencias Sociales. Documento de Internet. Visitado 24-11-2019.

Blaxter, L., Hughes, C. y Tight, M. (2010). Cómo se hace una investigación. Madrid: Editorial Gedisa.

Bonil, J., Calafell, G., Granados, J., Junyent, M. y Tarín, R. (2012). Un modelo formativo para avanzar en la ambientalización curricular. Profesorado. Revista de Currículum y Formación del Profesorado, 16(2), 145-163.

Bravo, W. (2011). Estudio de los conocimientos de los profesores sobre educación ambiental. Revista de Didáctica Ambiental, 9, 57-71.

Burgos, O. Gutiérrez, J. y Perales, F. (2012). La evaluación de la calidad en las ecoescuelas: un estudio comparado entre Chile y España. Revista Interciencia, 37(5), 340-347.

Burgos, 0. Perales, F. y Gutiérrez, J. (2010). Evaluación de la calidad de los establecimientos educativos incorporados al sistema nacional de certificación ambiental de la provincia de Bio-Bio (Chile). Profesorado. Revista de Currículum y Formación del Profesorado, 14(2), 213-240.

Calafell, G. y Bonil, J. (2014). Identificación y caracterización de las concepciones de medio ambiente de un grupo de profesionales de la educación ambiental. Revista Enseñanza de las Ciencias, 32(3), 205-225.

Calafell, G. y Junyent, M. (2017). La idea vector y sus esferas: una propuesta formativa para la ambientalización curricular desde la complejidad. Revista Teoría Educativa, 29(1), 189-216.

Camus, P. y Hajek, E. (1998). Historia Ambiental De Chile. Santiago de Chile: Andros Impresores.

Chopra, S. (2015). Integrating Environmental and Science Education: Issues and Possibilities. Educational Quest: An International Journal of Education and Applied Social Sciences, 6(2), 111-116. 
Correa, N. y Rodrigo, M. (2001). El cambio de perspectiva conceptual en las teorías implícitas sobre el medio ambiente. Revista Infancia y Aprendizaje, 24(4), 461-474.

Flechsig, K. y Schiefelbein, E. (2003). 20 Modelos Didácticos para América Latina. Washington, DC: Secretaría General de la Organización de Estados Americanos.

Gonzales, J. (2001). El paradigma interpretativo en la investigación social y educativa: nuevas respuestas para viejos interrogantes. Cuestiones pedagógicas: Revista de ciencias de la educación,15, 227-246.

Gonzales, R. y Lárez, J. (2009). Aproximación a los elementos para potenciar la Educación científica y la Educación ambiental: Reflexiones desde la praxis. Revista de Investigación, 33(68), 251-274.

Gutiérrez Pérez, J. , Pozo Llórente, T. y Fernández Cano, A. (2002). Los estudios de caso en la lógica de la investigación interpretativa. Revista Arbor, 171(675), 533-557.

Gutiérrez, J. y Pozo, T. (2006). Modelos teóricos contemporáneos y marcos de fundamentación de la educación ambiental para el desarrollo sostenible. Revista Iberoamericana de Educación, 41, 21-68.

Ministerio del Medio Ambiente (2009). Política Nacional de Educación para el Desarrollo Sustentable. Santiago de Chile. Documento de Internet. Visitado el $24-11$ - 2019

Mora, W. (2012). Educación en ciencias y educación ambiental: necesidad de una relación mutuamente beneficiosa. Revista EDUCyT, no Extraordinario. Diciembre.

Mosquera, C. (2011). La investigación sobre la formación de profesores desde la perspectiva del cambio didáctico. Magis, Revista Internacional de Investigación en Educación, 3(6), 265-282.

Muñoz, A. (2014). Environmental education in Chile, a pending task. Revista Ambiente \& Sociedade, 17(3),175-194.

Ponce, M. y Vega, G. (2009). Evaluación de la educación ambiental en la enseñanza secundaria municipal de Talca, Chile. Revista Neuma: Revista de Música y Docencia Musical, 2(2), 202-217.

Pujol, R. M. (2003). Didáctica de las ciencias en la educación primaria. Madrid: Editorial Síntesis.

Ripple, W. Wolf, C. Newsome, T. Galetti, M. Alamgir, M. Crist, E. and 15.364 scientist signatories from 184 countries. (2017). World scientists' warning to humanity: a second notice. BioScience, 67(12), 1026-1028.

Rivero, A., Martín del Pozo, R., Solís, E., Azcárate, P. y Porlán, R. (2017). Cambio del conocimiento sobre la enseñanza de las ciencias de futuros maestros. Revista Enseñanza de las Ciencias, 35(1), 29-52.

Rizoma (s.f.). Què és per a tu el medi? Barcelona: Rizoma Educació. Documento de Internet. Visita 24-11-2019. 
Rodríguez Marín, F., Fernández Arroyo, J. y García Díaz, J. (2014). Las hipótesis de transición como herramienta didáctica para la Educación Ambiental. Revista Enseñanza de las Ciencias, 32(3), 303-318.

Sauvé. L. (2004). Una cartografía de corrientes de educación ambiental. En M. Sato e I. Carvalho (Orgs.), A pesquisa em educação ambiental: cartografias de uma identidade narrativa em formação. Porto Alegre, Brasil: Artmed. Documento de Internet. Visita: 24-11-2019

Sauvé, L. (2010). Educación científica y educación ambiental: un cruce fecundo. Revista Enseñanza de las Ciencias, 28(1), 5-18.

Sauvé, L. (2013). Hacia una educación ecocientífica. Revista Tecné, Episteme y Didaxis, $34,7-12$.

Silva, E. (1996). Democracy, Market Economics, and Environmental Policy in Chile. Journal of interamerican studies and world affairs, 38(4), 1-33.

Squella, M. (2001). Environmental Education to Environmental Sustainability. Educational Philosophy and Theory, 33(2), 217-230.

Terrón, E. (2004). La educación ambiental en la educación básica, un proyecto inconcluso. Revista Latinoamericana de Estudios Educativos, 34(4), 107-164.

Torres, L., Benavides, J., Latoja, C. y Novoa, E. (2017). Presencia de una Educación Ambiental basada en conocimiento, actitudes y prácticas en la enseñanza de las ciencias naturales en establecimientos municipales de la ciudad de Los Ángeles, Chile. Revista Estudios Pedagógicos, 43(3), 311-323. 